\title{
Implicit large eddy simulation of compressible turbulence flow with $\mathrm{P}_{n} \mathrm{~T}_{m}-$ BVD scheme
}

\author{
Xi Deng ${ }^{\mathrm{a}, \mathrm{c}}$, Zhen-Hua Jiang ${ }^{\mathrm{b}, *}$, Feng Xiao ${ }^{\mathrm{a}}$, Chao Yan $^{\mathrm{b}}$ \\ a Department of Mechanical Engineering, Tokyo Institute of Technology, 2-12-1(i6-29) Ookayama, Meguro-ku, Tokyo 152-8550, Japan \\ ${ }^{\mathrm{b}}$ College of Aeronautics Science and Engineering, Beijing University of Aeronautics and Astronautics, Beijing 100191, PR China \\ ${ }^{\mathrm{c}}$ Aix Marseille University, CNRS, Centrale Marseille, Marseille M2P2, France
}

\section{A R T I C L E I N F O}

\section{Article history:}

Received 17 December 2018

Revised 21 May 2019

Accepted 11 July 2019

Available online 18 July 2019

\section{Keywords:}

Shock capturing

Compressible turbulence

Very high order schemes

$\mathrm{P}_{n} \mathrm{~T}_{m}$ - BVD scheme

WENO scheme

Implicit LES

\begin{abstract}
A B S T R A C T
Implicit large eddy simulation (ILES) of compressible turbulence with shock capturing schemes requires wide investigations and numerical experiments. In this study, a newly proposed $\mathrm{P}_{n} \mathrm{~T}_{m}$ - BVD (polynomial of $n$-degree and THINC function of $m$-level reconstruction based on BVD algorithm) shock capturing scheme is introduced to simulate compressible turbulence flow with ILES. The new scheme is designed by employing high-order linear-weight polynomials and THINC (Tangent of Hyperbola for INterface Capturing) functions with adaptive steepness as the reconstruction candidates. The final reconstruction function in each cell is determined with a multi-stage BVD (Boundary Variation Diminishing) algorithm so as to effectively control numerical oscillation and dissipation. Numerical tests involving shock waves and broadband turbulence are conducted in comparison with WENO (Weighted Essentially Non-oscillatory) schemes which are widely used in ILES. The results demonstrate performing ILES with $\mathrm{P}_{n} \mathrm{~T}_{m}-\mathrm{BVD}$ scheme is able to obtain higher resolution and more faithful results than WENO does. Importantly, the superiority of $\mathrm{P}_{n} \mathrm{~T}_{m}$ - BVD becomes more notable in high wave-number region. Thus this paper provides and verifies a new scheme which is promising in providing high-resolution results for real-case ILES of compressible turbulence flow.
\end{abstract}

\section{Introduction}

Having been devoted tremendous efforts in the past decades, computational fluid dynamics have reached a mature stage for many engineering applications. However, there are still some difficult issues that are of great application importance but remain unsolved yet. Numerical simulation of compressible turbulence is among them. The numerical schemes designed for the shock-turbulence simulation require schemes being low-dissipative to resolve small-scale structure but also being able to obtain stable solution involving strong shock.

The popularly used practical methods, such as high-resolution shock capturing TVD (Total Variation Diminishing) [1,2], ENO (Essentially Non-oscillatory) [3-5] and WENO (Weighted Essentially Non-oscillatory) [6,7] methods are found to be too dissipative to adequately resolve small flow structures that play essential role in turbulent interaction processes [8]. Introducing central flux which is preferable for turbulence flow simulations, hybrid WENO/central schemes [9-13] are able to

\footnotetext{
* Corresponding author.

E-mail address: jiangzhenhua@buaa.edu.cn (Z.-H. Jiang).
} 
resolve broadband turbulence. However, the performance of hybrid schemes usually rely on shock sensors. Artificial viscosity/diffusivity methods [14-17] generally give better resolution than WENO schemes. Still, the introduced artificial viscosity may also overwhelm small-scale structures.

The excessive numerical dissipation of high order shock-capturing schemes comes intrinsically from the dilemma that one has to compromise between high-order interpolation and spurious oscillation in the current paradigm based on polynomial reconstruction and nonlinear limiting projection. Known as the Godunov barrier, any reconstruction using polynomial higher than 2nd order tends to generate numerical oscillations in presence of discontinuity or large gradient. On one hand, a high-order polynomial is preferred for smooth solution, on the other hand a lower order (or smoother) interpolant is suggested in the vicinity of discontinuous solution. It is realized automatically through a nonlinear switching based on smoothness measurement. Being the most representative numerical method of this sort, the WENO reconstruction is designed so as to retrieve the highest possible convergence rate in the smooth region while degrade to a flatter or lower-order interpolant around discontinuous solution. In spite of huge amount of work devoted to devise nonlinear limiting formulations based on WENO concept, the existing schemes show at least the following two drawbacks. (1) The nonlinearly weighted reconstruction functions can hardly retrieve the unlimited polynomials that possess the desired numerical properties for smooth solutions, and (2) for high-order reconstructions using polynomials with nonlinear weights might not be adequately robust nor effectively suppress numerical oscillations in the presence of strong discontinuities.

Besides the most representative WENO methodology, other shock-capturing schemes equipped with nonlinear limiters have also been proposed in [18-21]. For example, the high-order Monotonicity-Preserving (MP) scheme which can effectively suppress numerical oscillations across the discontinuity and preserve the accuracy at extrema is first designed in [20]. The MP scheme is further improved by the work $[22,23]$ to optimize the dispersion and dissipation property of MP scheme. Although the MP schemes show some advantages over WENO schemes, the MP limiter may be still triggered in high wavenumber region, which undermines the accuracy of underlying low-dissipation interpolation. Being a different approach from finite volume method (FVM) or finite difference method (FDM), discontinuous Galerkin (DG) method [24-26] introduces local high order reconstruction functions by adding degrees of freedom in the local cell element. Limiting processes are also demanded to realize shock capturing [27-29]. Designing such a limiting process is still a hot topic in the community of high order schemes [30].

Although shock capturing schemes have inherent numerical dissipation of which sources usually come from upwind biased interpolation and limiting processes, shock capturing schemes are still used in compressible turbulence simulations through an approach called implicit large eddy simulation (ILES) [31]. Originated from the observations in [32], ILES is one of simple and efficient way to simulate compressible turbulence flow. Different from direct numerical simulation (DNS) which requires an expensive condition to solve Navier-Stokes (NS) equation and also different from explicit LES method [33] which requires additional sub-grid scale (SGS) model resulted from filtering operation of NS equation, ILES method solves NS equation with dissipated non-oscillatory finite volume (NFV) numerical schemes. In ILES, the embedded numerical dissipation in NFV schemes is used in replace of the explicit SGS models. It has been shown in the work [34] that ILES can give results close to those from strict DNS or explict LES. However, as indicated in [35,36], ILES methods need to be carefully validated and understanding of these methods still relies on performing numerical experiments. For example, the work of [37] shows that numerical dissipation of WENO schemes can play a similar role as a very disspative SGS model. In the work [38], performance of different high order WENO scheme on ILES has been investigated on compressible turbulence flow and turbulent combustion. In [36], ILES has been studied with very high order WENO schemes (up to eleventh order), which indicates that a higher order scheme provides a more pragmatic approach than using a lower order scheme.

We have recently proposed a new reconstruction approach for Godunov finite volume method, so-called BVD method, to resolve both smooth and discontinuous solutions with high fidelity. A BVD method adaptively chooses the interpolant from the BVD-admissible reconstruction functions so that the difference between the reconstructed values at cell boundary is minimized, which then effectively reduces the numerical dissipation. Under the BVD concept, a new class of schemes can be designed, using proper BVD algorithms and BVD-admissible reconstruction functions [39-42]. Our previous works show that the BVD schemes are able to substantially improves the numerical solutions, particularly for discontinuous and small flow structures. For example, it has been shown in [41] that BVD algorithm can prevent spurious phenomena which may appear with stiff detonation waves. In our recent work [43,44], high-order unlimited polynomials and THINC function are used as the candidate reconstruction functions based on a multi-stage BVD algorithm. The resultant schemes can retrieve the numerical dispersion and dissipation properties of the unlimited polynomials for all wave numbers, thus demonstrate superior solution quality for smooth solutions. Meanwhile, the spurious oscillation can be effectively eliminated through the BVD algorithm that properly chooses the THINC function for reconstruction in the neighboring cells of a discontinuity. The extensive numerical verification for scalar hyperbolic conservation law and inviscid Euler equations reveal that the BVD schemes using unlimited high-order polynomials have more appealing numerical properties in comparison with other existing high resolution schemes that make use of nonlinear limiting projections. These methods thus have a great potential to simulate turbulent flows with ILES. In this paper, we implement the BVD schemes to compressible Navier-Stokes equations to evaluate their performance in ILES of compressible turbulence flows.

The rest of this paper is organized as follows. Section 2 provides an outline of Godunov finite volume formulation. Section 3 describes the multi-stage BVD scheme that use the unlimited high-order polynomials and THINC as the candidate reconstruction function. Section 4 presents numerical tests for the proposed schemes. Special attention is paid to a set of benchmark tests of compressible Navier Stokes equations for turbulent flows of different Reynolds numbers. It is observed 
that performing ILES with the present BVD schemes are superior to the WENO scheme in terms of reproducing turbulent statistic properties. We end the paper with some concluding remarks, in Section 5.

\section{Finite volume method}

To simulate compressible viscous flow, the compressible Navier-Stokes equations for a calorically perfect gas are solved:

$$
\begin{aligned}
& \frac{\partial \rho}{\partial t}+\nabla \cdot \mathbf{m}=0 \\
& \frac{\partial \mathbf{m}}{\partial t}+\nabla \cdot(\mathbf{m} \otimes \mathbf{u}+p \delta)=\nabla \cdot \tau, \\
& \frac{\partial E}{\partial t}+\nabla \cdot(\mathbf{u} E+p \mathbf{u})=\nabla \cdot(\mathbf{u} \cdot \tau-\mathbf{q}),
\end{aligned}
$$

where $\rho$ is the density, $\mathbf{m}$ is the momentum, $\mathbf{u}$ is the velocity, $\mathbf{p}$ is the pressure, $\delta$ is the unit tensor, $E$ is the total energy, $\tau$ is the viscous stress tensor and $\mathbf{q}$ is the heat flux. To closure the above equation system, the following equation of state is applied:

$$
p=(\gamma-1) \rho e
$$

in which $\gamma$ is the ratio of specific heats and $e$ is the specific internal energy. According to Stokes' hypothesis for a Newtonian fluid, the viscous stress tensor is calculated as

$$
\tau=2 \mu \mathbf{S}-\frac{2}{3} \mu(\nabla \cdot \mathbf{u})
$$

where strain rate tensor $\mathbf{S}$ is defined as $\mathbf{S}=\frac{1}{2}\left(\nabla \mathbf{u}+(\nabla \mathbf{u})^{T}\right)$ and $\mu$ is the dynamic shear viscosity. Following Fourier's law, the heat flux is defined as $\mathbf{q}=-k \nabla T$ where $k$ is the thermal conductivity and $T$ is the temperature. It has been long assumed that the accuracy of the convective terms is critical for resolving the turbulence [45]. Thus, the main challenge to simulate shock-turbulence problem is to introduce a high accuracy scheme to discretize the Euler equation systems which is recovered from Navier-Stokes by ignoring viscous effect. The Euler equation systems can be further analyzed by solving the one-dimensional scalar model equation as

$$
\frac{\partial q}{\partial t}+\frac{\partial f(q)}{\partial x}=0
$$

where $q$ is the solution variables which can be in characteristic space and $f(q)$ is the flux function.

Considering 1D scalar model equation of Eq. (6), a standard finite volume method is applied to obtain the numerical solutions. We divide the computational domain into $N$ non-overlapping cell elements, $\mathcal{I}_{i}: x \in\left[x_{i-1 / 2}, x_{i+1 / 2}\right], i=1,2, \ldots, N$, with a uniform grid spacing $h=\Delta x=x_{i+1 / 2}-x_{i-1 / 2}$. the volume-integrated average value $\bar{q}_{i}(t)$ in cell $\mathcal{I}_{i}$ is defined as

$$
\bar{q}_{i}(t) \approx \frac{1}{\Delta x} \int_{x_{i-1 / 2}}^{x_{i+1 / 2}} q(x, t) d x .
$$

The semi-discrete version of Eq. (6) in the finite volume form can be expressed as an ordinary differential equation (ODE)

$$
\frac{\partial \bar{q}(t)}{\partial t}=-\frac{1}{\Delta x}\left(\tilde{f}_{i+1 / 2}-\tilde{f}_{i-1 / 2}\right),
$$

where the numerical fluxes $\tilde{f}$ at cell boundaries can be computed by a Riemann solver

$$
\tilde{f}_{i+1 / 2}=f_{i+1 / 2}^{\text {Riemann }}\left(q_{i+1 / 2}^{L}, q_{i+1 / 2}^{R}\right)
$$

as long as the reconstructed left-side value $q_{i+1 / 2}^{L}$ and right-side value $q_{i+1 / 2}^{R}$ at cell boundaries are provided. Essentially, the Riemann flux can be written in a canonical form as

$$
\left.f_{i+1 / 2}^{\text {Riemann }}\left(q_{i+1 / 2}^{L}, q_{i+1 / 2}^{R}\right)=\frac{1}{2}\left(f\left(q_{i+1 / 2}^{L}\right)+f\left(q_{i+1 / 2}^{R}\right)\right)-\frac{\left|a_{i+1 / 2}\right|}{2}\left(q_{i+1 / 2}^{R}-q_{i+1 / 2}^{L}\right)\right),
$$

where $a_{i+1 / 2}$ stands for the characteristic speed of the hyperbolic conservation law. The remaining main task is how to calculate $q_{i+1 / 2}^{L}$ and $q_{i+1 / 2}^{R}$ through the reconstruction process.

\section{3. $P_{n} T_{m}$ - BVD: a new reconstruction scheme}

In this subsection, we give the details of how to calculate $q_{i+1 / 2}^{L}$ and $q_{i+1 / 2}^{R}$ using the newly proposed $\mathrm{P}_{n} \mathrm{~T}_{m}-\mathrm{BVD}$ schemes in [43]. These schemes are designed by employing linear weight polynomial of $n$-degree and THINC function of $m$-level as the candidate interpolants. The final reconstruction function in each cell is selected from these candidate interpolants with the BVD algorithm. Next, we introduce the candidate interpolants before the description of the BVD algorithm. 


\subsection{Candidate interpolant $P_{n}$ : linear upwind scheme of $\mathrm{n}$-degree polynomial}

A finite volume scheme of $(n+1)$ th order can be constructed from a spatial approximation for the solution in the target cell $\mathcal{I}_{i}$ with a polynomial $\tilde{q}_{i}^{\text {Pn }}(x)$ of degree $n$. The $n+1$ unknown coefficients of the polynomial are determined by requiring that $\tilde{q}_{i}^{P n}(x)$ has the same cell average on each cell over an appropriately selected stencil $S=\left\{i-n^{-}, \ldots, i+n^{+}\right\}$with $n^{-}+$ $n^{+}=n$, which is expressed as

$$
\frac{1}{\Delta x} \int_{x_{j-1 / 2}}^{x_{j+1 / 2}} \tilde{q}_{i}^{P n}(x) d x=\bar{q}_{j}, \quad j=i-n^{-}, i-n^{-}+1, \ldots, i+n^{+} .
$$

To construct $2 r-1$ order upwind-biased (UW) finite volume schemes as detailed in [6,7,46], the stencil is defined with $n^{-}=$ $n^{+}=r-1$. The unknown coefficients of polynomial of $2 r-2$ degree can be then calculated from (11). With the polynomial $\tilde{q}_{i}^{\text {Pn }}(x)$, high order approximation for reconstructed values at the cell boundaries can be obtained by

$$
q_{i+\frac{1}{2}}^{L, P n}=\tilde{q}_{i}^{P n}\left(x_{i+\frac{1}{2}}\right) \text { and } q_{i-\frac{1}{2}}^{R, P n}=\tilde{q}_{i}^{P n}\left(x_{i-\frac{1}{2}}\right) .
$$

The analysis of $[6,7,46]$ shows that in smooth region, the approximation with polynomial (12) can achieve $2 r-1$ order accuracy. In this work, we apply schemes from fifth order $(r=3)$ to ninth order $(r=5)$ by using polynomials of 4 th, 6th, and 8th degree as underlying scheme for smooth solution. The explicit formulas of $q_{i+1 / 2}^{L, P n}$ and $q_{i-1 / 2}^{R, P n}$ for ( $\left.\mathrm{n}+1\right)$ th-order scheme are given as follows:

- 5th-order scheme

$$
\begin{aligned}
& q_{i+1 / 2}^{L, P 4}=\frac{1}{30} \bar{q}_{i-2}-\frac{13}{60} \bar{q}_{i-1}+\frac{47}{60} \bar{q}_{i}+\frac{9}{20} \bar{q}_{i+1}-\frac{1}{20} \bar{q}_{i+2}, \\
& q_{i-1 / 2}^{R, P 4}=\frac{1}{30} \bar{q}_{i+2}-\frac{13}{60} \bar{q}_{i+1}+\frac{47}{60} \bar{q}_{i}+\frac{9}{20} \bar{q}_{i-1}-\frac{1}{20} \bar{q}_{i-2} .
\end{aligned}
$$

- 7th-order scheme

$$
\begin{aligned}
& q_{i+1 / 2}^{L, P 6}=-\frac{1}{140} \bar{q}_{i-3}+\frac{5}{84} \bar{q}_{i-2}-\frac{101}{420} \bar{q}_{i-1}+\frac{319}{420} \bar{q}_{i}+\frac{107}{210} \bar{q}_{i+1}-\frac{19}{210} \bar{q}_{i+2}+\frac{1}{105} \bar{q}_{i+3}, \\
& q_{i-1 / 2}^{R, P 6}=-\frac{1}{140} \bar{q}_{i+3}+\frac{5}{84} \bar{q}_{i+2}-\frac{101}{420} \bar{q}_{i+1}+\frac{319}{420} \bar{q}_{i}+\frac{107}{210} \bar{q}_{i-1}-\frac{19}{210} \bar{q}_{i-2}+\frac{1}{105} \bar{q}_{i-3} .
\end{aligned}
$$

- 9th-order scheme

$$
\begin{aligned}
q_{i+1 / 2}^{L, P 8}= & \frac{1}{630} \bar{q}_{i-4}-\frac{41}{2520} \bar{q}_{i-3}+\frac{199}{2520} \bar{q}_{i-2}-\frac{641}{2520} \bar{q}_{i-1}+\frac{1879}{2520} \bar{q}_{i} \\
& +\frac{275}{504} \bar{q}_{i+1}-\frac{61}{504} \bar{q}_{i+2}+\frac{11}{501} \bar{q}_{i+3}-\frac{1}{504} \bar{q}_{i+4}, \\
q_{i-1 / 2}^{R, P 8}= & \frac{1}{630} \bar{q}_{i+4}-\frac{41}{2520} \bar{q}_{i+3}+\frac{199}{2520} \bar{q}_{i+2}-\frac{641}{2520} \bar{q}_{i+1}+\frac{1879}{2520} \bar{q}_{i} \\
& +\frac{275}{504} \bar{q}_{i-1}-\frac{61}{504} \bar{q}_{i-2}+\frac{11}{501} \bar{q}_{i-3}-\frac{1}{504} \bar{q}_{i-4} .
\end{aligned}
$$

\subsubsection{Candidate interpolant $T_{m}$ : non-polynomial THINC function with $\mathrm{m}$-level steepness}

Another candidate interpolation function in $\mathrm{P}_{n} \mathrm{~T}_{m}-\mathrm{BVD}$ scheme makes use of the THINC interpolation which is a differentiable and monotone Sigmoid function [47,48]. The piecewise THINC reconstruction function is written as

$$
\tilde{q}_{i}^{T}(x)=\bar{q}_{\min }+\frac{\bar{q}_{\max }}{2}\left[1+\theta \tanh \left(\beta\left(\frac{x-x_{i-1 / 2}}{x_{i+1 / 2}-x_{i-1 / 2}}-\tilde{x}_{i}\right)\right)\right],
$$

where $\bar{q}_{\min }=\min \left(\bar{q}_{i-1}, \bar{q}_{i+1}\right), \bar{q}_{\max }=\max \left(\bar{q}_{i-1}, \bar{q}_{i+1}\right)-\bar{q}_{\min }$ and $\theta=\operatorname{sgn}\left(\bar{q}_{i+1}-\bar{q}_{i-1}\right)$. The jump thickness is controlled by the parameter $\beta$, i.e. a small value of $\beta$ leads to a smooth profile while a large one leads to a sharp jump-like distribution. The unknown $\tilde{x}_{i}$, which represents the location of the jump center, is computed from constraint condition $\bar{q}_{i}=\frac{1}{\Delta x} \int_{x_{i-1 / 2}}^{x_{i+1 / 2}} \tilde{q}_{i}^{T}(x) d x$.

Since the value given by hyperbolic tangent function $\tanh (x)$ lays in the region of $[-1,1]$, the value of THINC reconstruction function $\tilde{q}_{i}^{T}(x)$ is rigorously bounded by $\bar{q}_{i-1}$ and $\bar{q}_{i+1}$. Given the reconstruction function $\tilde{q}_{i}^{T}(x)$, we calculate the boundary values $q_{i+1 / 2}^{L, T}$ and $q_{i-1 / 2}^{R, T}$ by $q_{i+1 / 2}^{L, T}=\tilde{q}_{i}^{T}\left(x_{i+1 / 2}\right)$ and $q_{i-1 / 2}^{R, T}=\tilde{q}_{i}^{T}\left(x_{i-1 / 2}\right)$, respectively.

Following [42], in this work we use THINC functions with $\beta$ of $m$-level to represent different steepness, to realize nonoscillatory and less-dissipative reconstructions adaptively for various flow structures. A THINC reconstruction function $\tilde{q}_{i}^{T k}(x)$ with $\beta_{k}$ gives the reconstructed values $q_{i+1 / 2}^{L, T k}$ and $q_{i-1 / 2}^{R, T k},(k=1,2, \ldots, m)$. We will use $m$ up to three in present study. 


\subsection{The BVD algorithm}

In the $\mathrm{P}_{n} \mathrm{~T}_{m}$ - BVD schemes, reconstruction function is determined from the candidate interpolants with $k$-stage $(k=m)$ BVD algorithm so as to minimize the TBV of the target cell. We denote the reconstruction function in the target cell $\mathcal{I}_{i}$ after the $k$ th stage BVD as $\tilde{q}_{i}^{<k>}(x)$.

The $k$-stage BVD algorithm is formulated as follows:

(I): Initial stage $(k=0)$ :

(I-I) As the first step, use the linear high-order upwind scheme as the base reconstruction scheme and initialize the reconstructed function as $\tilde{q}_{i}^{<0>}(x)=\tilde{q}_{i}^{P n(x)}$.

(II) The intermediate BVD stage $(k=1, \ldots, m-1)$ :

(II-I) Set $\tilde{q}_{i}^{<k>}(x)=\tilde{q}_{i}^{<k-1>}(x)$

(II-II) Calculate the TBV values for target cell $\mathcal{I}_{i}$ from the reconstruction of $\tilde{q}_{i}^{<k>}(x)$

$$
T B V_{i}^{<k>}=\left|q_{i-1 / 2}^{L,<k>}-q_{i-1 / 2}^{R,<k>}\right|+\left|q_{i+1 / 2}^{L,<k>}-q_{i+1 / 2}^{R,<k}\right|
$$

and from the THINC function $\tilde{q}_{i}^{T k}(x)$ with a steepness $\beta_{k}$ as

$$
T B V_{i}^{T k}=\left|q_{i-1 / 2}^{L, T k}-q_{i-1 / 2}^{R, T k}\right|+\left|q_{i+1 / 2}^{L, T k}-q_{i+1 / 2}^{R, T k}\right| .
$$

(II-III) Modify the reconstruction function for cells $i-1$, $i$ and $i+1$ according to the following BVD algorithm

$$
\tilde{q}_{j}^{<k>}(x)=\tilde{q}_{j}^{T k}(x), j=i-1, i, i+1 ; \quad \text { if } T B V_{i}^{T k}<T B V_{i}^{<k>} .
$$

(III) The final BVD stage $(k=m)$ :

(III-I) Given the reconstruction functions $\tilde{q}_{i}^{<m-1>}(x)$ from above stage, compute the TBV using the reconstructed cell boundary values from previous stage by

$$
T B V_{i}^{<m-1>}=\left|q_{i-1 / 2}^{L,<m-1>}-q_{i-1 / 2}^{R,<m-1>}\right|+\left|q_{i+1 / 2}^{L,<m-1>}-q_{i+1 / 2}^{R,<m-1>}\right|,
$$

and the TBV for THINC function of $\beta_{m}$ by

$$
T B V_{i}^{T m}=\left|q_{i-1 / 2}^{L, T m}-q_{i-1 / 2}^{R, T m}\right|+\left|q_{i+1 / 2}^{L, T m}-q_{i+1 / 2}^{R, T m}\right| .
$$

(III-II) Determine the final reconstruction function for cell $\mathcal{I}_{i}$ using the BVD algorithm as

$$
\tilde{q}_{i}^{<m>}(x)= \begin{cases}\tilde{q}_{i}^{T m} ; & \text { if } T B V_{i}^{T m}<T B V_{i}^{<m-1>}, \\ \tilde{q}_{i}^{<m-1>} ; & \text { otherwise }\end{cases}
$$

(III-III) Compute the reconstructed values on the left-side of $x_{i+\frac{1}{2}}$ and the right-side of $x_{i-\frac{1}{2}}$ respectively by

$$
q_{i+\frac{1}{2}}^{L}=\tilde{q}_{i}^{<m>}\left(x_{i+\frac{1}{2}}\right) \text { and } q_{i-\frac{1}{2}}^{R}=\tilde{q}_{i}^{<m>}\left(x_{i-\frac{1}{2}}\right) .
$$

In this study, we propose and test fifth order scheme with $\mathrm{P}_{4} \mathrm{~T}_{2}-\mathrm{BVD}$, seventh order scheme with $\mathrm{P}_{6} \mathrm{~T}_{3}-\mathrm{BVD}$ and ninth order scheme with $\mathrm{P}_{8} \mathrm{~T}_{3}-\mathrm{BVD}$. According to previous study in [42], in all tests of the present study we use $\beta_{1}=1.1$ and $\beta_{2}=1.8$ for $\mathrm{P}_{4} \mathrm{~T}_{2}-\mathrm{BVD}$, and $\beta_{1}=1.2, \beta_{2}=1.1$ and $\beta_{3}=1.8$ for $\mathrm{P}_{n} \mathrm{~T}_{3}-\mathrm{BVD}(n=6,8)$ schemes.

Remark 1. In current schemes, simple upwind-biased interpolation functions are employed as underlying high order schemes. However, less-dissipative interpolations optimized as [23,49] are also possible to be employed as underlying schemes.

\section{Numerical experiments}

In this section, we show numerical results for benchmark tests to verify the performance of $\mathrm{P}_{n} \mathrm{~T}_{m}-\mathrm{BVD}$ in simulating compressible turbulence. We will firstly analyze the spectral property of schemes and solve non-broadband 1D Euler equations in Sections 4.2 and 4.3. Then the simulation results of three-dimensional broadband compressible turbulence problems will be given. All numerical results are compared with those of the mapped WENO (WENOM) [50] schemes. Although there are other improved WENO schemes such as WENOZ [51], it is shown in [38] that the performances of WENOM and WENOZ are almost equivalent. The CFL number 0.4 is used in our tests unless specifically noted. 

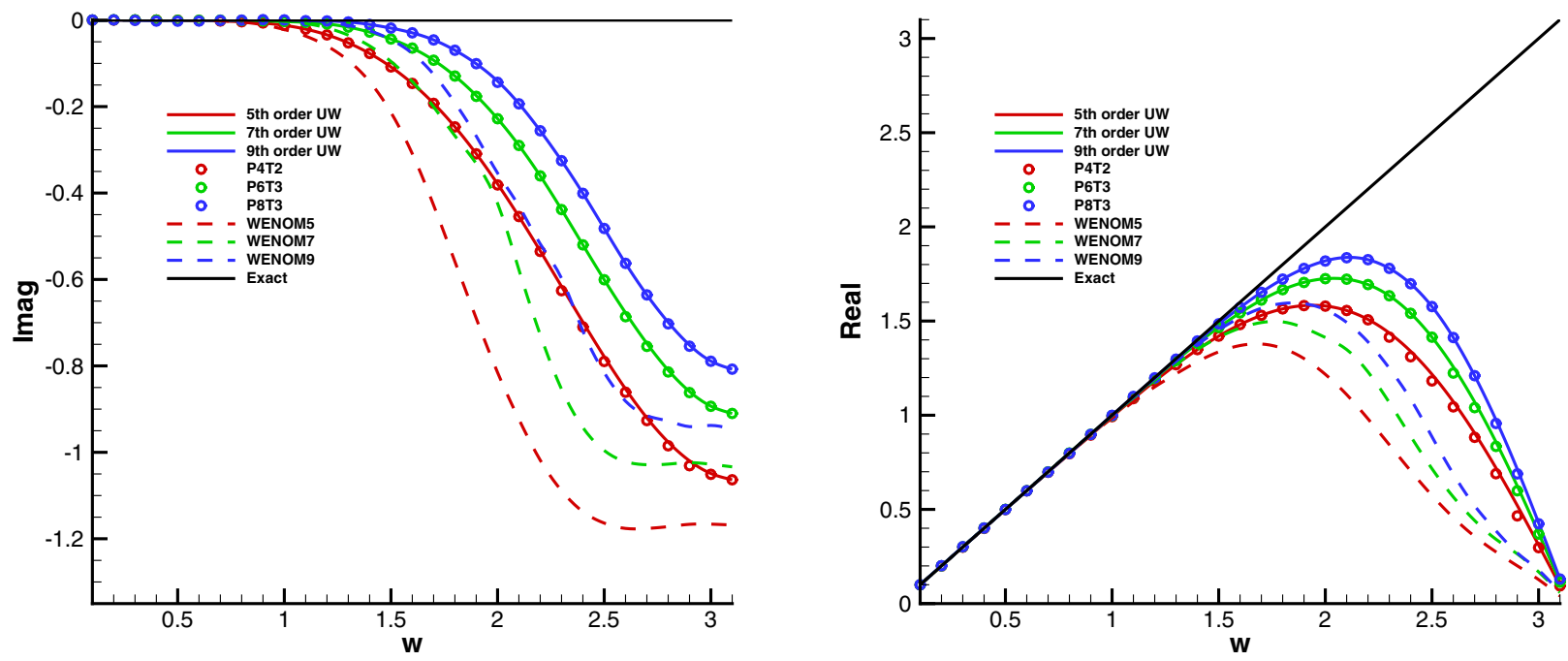

Fig. 1. Approximate dispersion and dissipation properties for different schemes. Imaginary parts of modified wavenumber are shown in the left panel, while real parts are shown in the right. Comparisons between linear upwind schemes, WENOM and $\mathrm{P}_{n} \mathrm{~T}_{m}-\mathrm{BVD}_{\mathrm{s}}$ schemes.

\subsection{Spectral property}

We analyzed the spectral property of the proposed scheme by using the approximate dispersion relation (ADR) method described in [52]. The numerical dissipation and dispersion of a scheme can be evaluated respectively from the imaginary and real parts of the modified wavenumber.

The spectral properties of the high order linear upwind schemes, WENOM and $\mathrm{P}_{n} \mathrm{~T}_{m}-\mathrm{BVD}$ schemes are shown in Fig. 1 . The WENOM schemes show significant discrepancies with the linear scheme for high wavenumber regime. It is partly owed to the fact that the WENO smoothness indicators tend to mis-interpret among high-wavenumber structures and discontinuities. Thus the numerical dissipation introduced by the wrongly triggered WENO nonlinear adaptation may smear out the high-wavenumber structures which are common in turbulence flow. On the contrary, the newly proposed $\mathrm{P}_{n} \mathrm{~T}_{m}-\mathrm{BVD}$ schemes have almost the same spectral property as their underlying linear schemes, which surpasses WENO methodology. Thus the $\mathrm{P}_{n} \mathrm{~T}_{m}-\mathrm{BVD}$ can distinguish high-wavenumber structures from true discontinuities, and preserve the lowdissipation property of high order upwind schemes. The spectral property indicates that $\mathrm{P}_{n} \mathrm{~T}_{m}-\mathrm{BVD}$ schemes are more preferable than WENOM in simulating smooth flow region.

\subsection{Inviscid strong Lax's problem}

In shock-turbulence simulations, schemes should be able to deal with the possibility of existing strong discontinuities such as shock waves and detonation waves [53-58]. Thus, we conduct a Lax problem to test the robustness of different schemes on capturing strong discontinuities. The initial condition is prescribed as

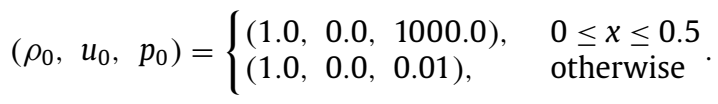

With initial high pressure ratio, a right-moving Mach 198 shock and a contact is generated. The computation lasts until time $t=0.012$ with 200 cell elements. The numerical solutions from different schemes for density field are plotted in Fig. 2 . In each figures, the zoomed regions around the highly compressed region are also presented. We compare $\mathrm{P}_{n} \mathrm{~T}_{m}-\mathrm{BVD}_{\mathrm{D}}$ with WENOM schemes at the same order. It is obvious that $\mathrm{P}_{n} \mathrm{~T}_{m}-$ BVD schemes produce more faithful solution with reduced errors in both dissipation and overshooting. Around discontinuities, WENOM schemes result in more diffusive profiles. More seriously, WENOM schemes produce overshoot values in highly compressed region. As order of WENOM increased, the overshoot and numerical oscillations become more obvious. This test justifies the proposed scheme as a robust solver for strong discontinuities with high accuracy. We also observe that $\mathrm{P}_{n} \mathrm{~T}_{m}-\mathrm{BVD}$ schemes of different orders produce similar results across the contact where the THINC function is selected by the BVD algorithm. It is noteworthy that the number of cells required to capture the discontinuity is independent of grid resolutions.

\subsection{Inviscid shock density wave interaction problem}

In order to verify the performance of the schemes in capturing shocks as well as smooth solutions of different scales, we simulated the case proposed in [59], which serves as a good and simple test bed for the simulations of compressible 


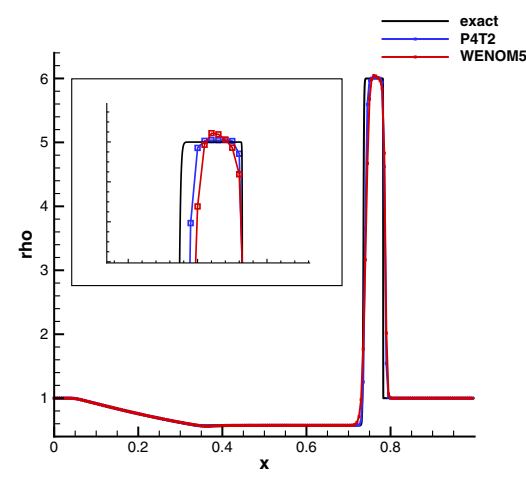

(a) 5th order scheme

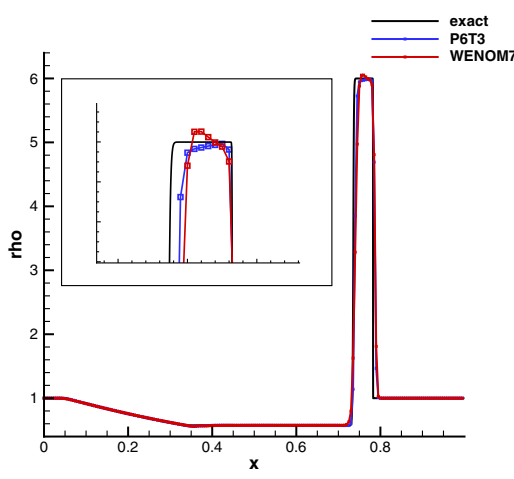

(b) 7 th order scheme

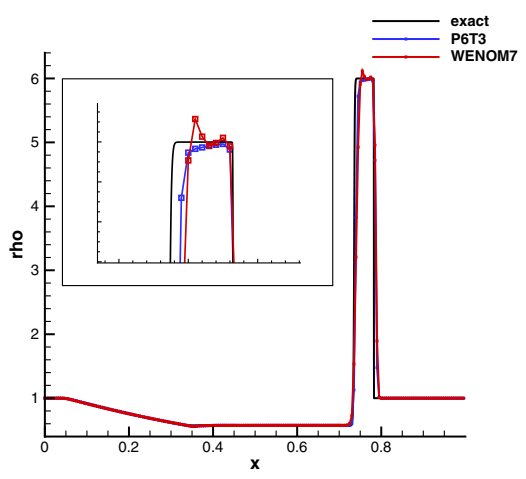

(c) 9 th order scheme

Fig. 2. Numerical results of strong Lax's problem with Mach 198 for density field at time $t=0.012$ with 200 cells. Comparisons are made between WENOM and $\mathrm{P}_{n} \mathrm{~T}_{m}-\mathrm{BVD}$ at the same order.

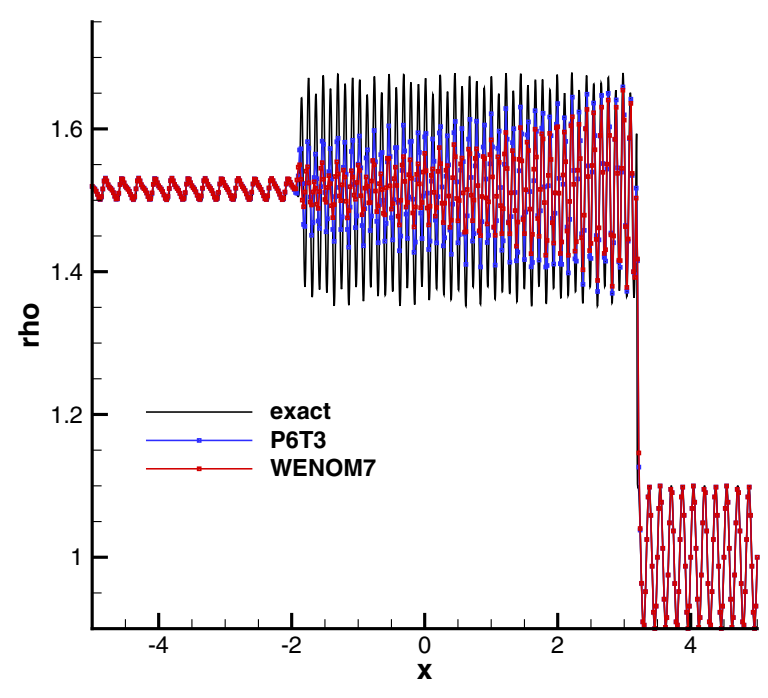

(a) 7 th order scheme

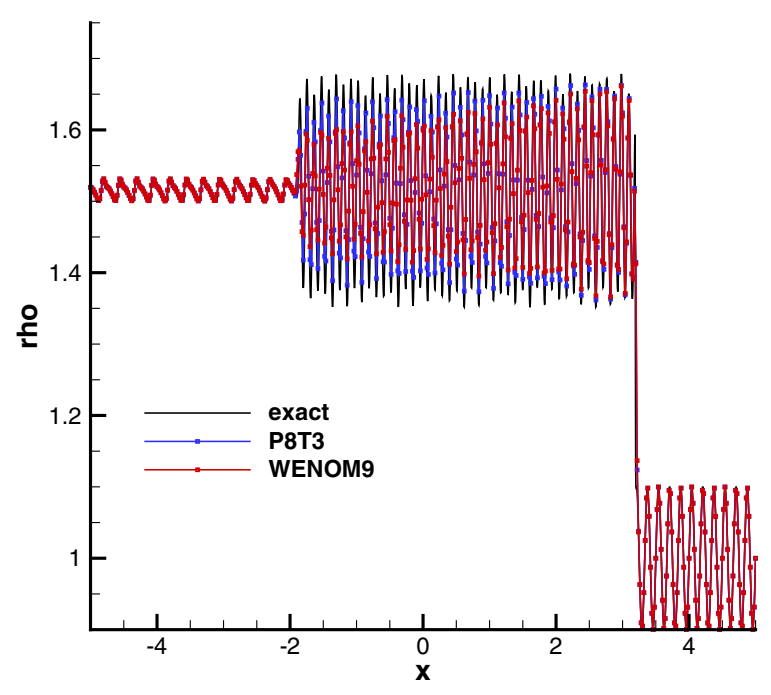

(b) 9th order scheme

Fig. 3. Numerical results of shock density wave interaction problem. Comparisons between 7 th order WENOM and $\mathrm{P}_{6} \mathrm{~T}_{3}$ are shown in the left panel, and between 9th order WENOM and $\mathrm{P}_{8} \mathrm{~T}_{3}$ and $\mathrm{P}_{10} \mathrm{~T}_{3}$ in the right panel.

turbulence involving shock waves. In this case, a shock wave interacts with density disturbances and generates a flow field containing waves of higher wavenumber and discontinuities. The initial condition is specified similarly as [59]

$$
\left(\rho_{0}, u_{0}, p_{0}\right)= \begin{cases}(1.515695,0.523346,1.805), & \text { if } x \leq-4.5 \\ (1+0.1 \sin (12 \pi x), 0,1), & \text { otherwise }\end{cases}
$$

The computation was carried out up to $t=5.0$. The numerical solutions with 500 cells were shown in Figs. 3 and 4 with zoomed region, where comparisons between WENOM and $\mathrm{P}_{n} \mathrm{~T}_{m}-\mathrm{BVD}$ at different order are included. It is obvious that the $\mathrm{P}_{n} \mathrm{~T}_{m}$ - BVD schemes resolve better density perturbations and capture more accurately the peak of the waves. Also, the resolution of high-frequency waves is improved with the order of the underlying polynomial increased. This test confirms that $\mathrm{P}_{n} \mathrm{~T}_{m}-\mathrm{BVD}$ schemes can simultaneously resolve flows containing both smooth and discontinuous regions with high accuracy.

\subsection{Inviscid Taylor-Green vortex}

The numerical dissipation of the schemes are further evaluated through an inviscid Taylor-Green vortex problem $[8,60,61]$. Same as [8], the initial condition is given by 


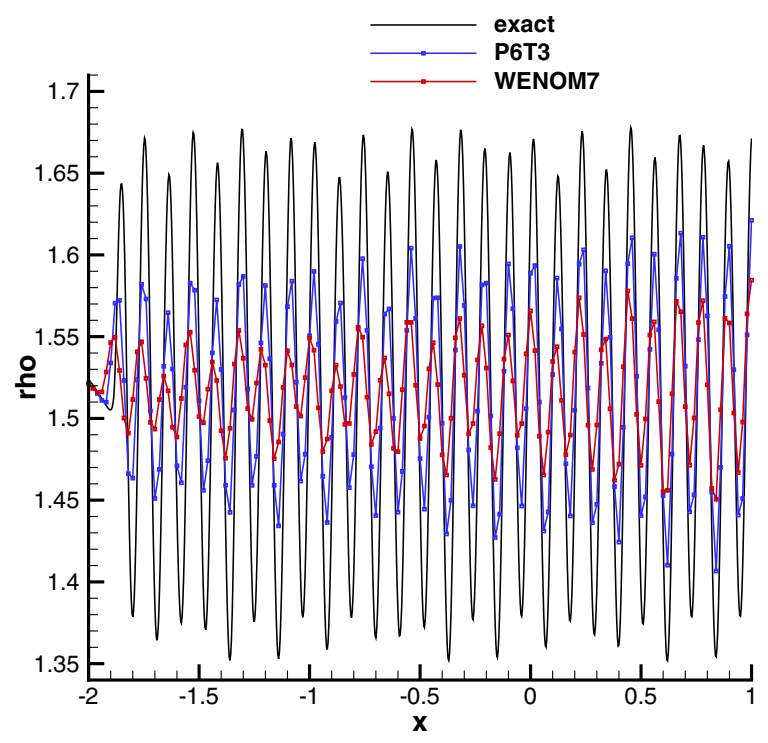

(a) 7 th order scheme

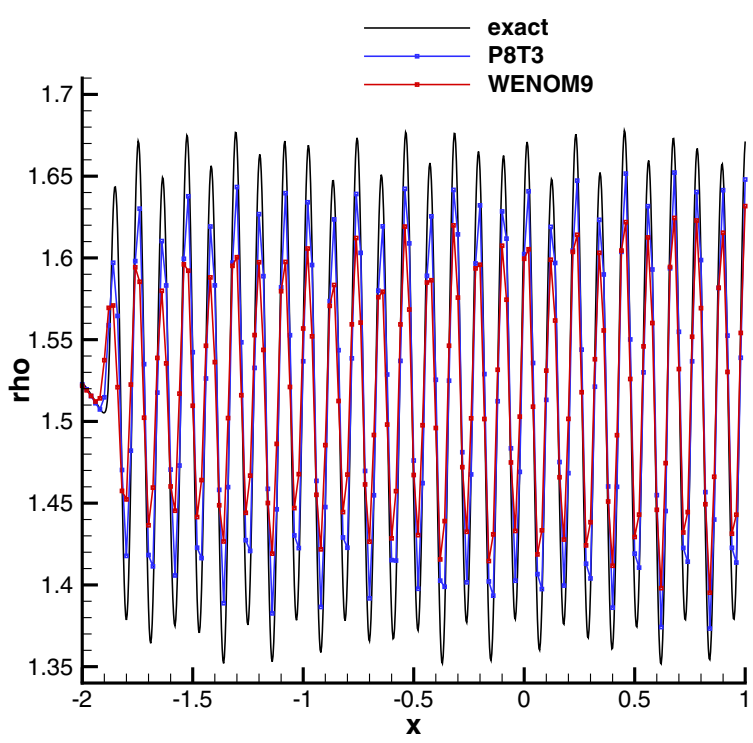

(b) 9 th order scheme

Fig. 4. The same as Fig. 3 but the zoomed region is shown.

$$
\begin{aligned}
\rho & =1, \\
u & =\sin (x) \cos (y) \cos (z), \\
v & =-\cos (x) \sin (y) \cos (z), \\
w & =0, \\
p & =100+\frac{[\cos (2 x)+\cos (2 y)][\cos (2 z)+2]-2}{16}
\end{aligned}
$$

where the pressure is high enough to make the problem nearly incompressible. The domain is $[0,2 \pi)^{3}$ with uniform grid size of $2 \pi / 64$ in each direction. Periodic boundary condition is prescribed for all boundaries. Comparisons are made between the newly proposed $\mathrm{P}_{n} \mathrm{~T}_{m}-\mathrm{BVD}$ and WENOM schemes. Firstly, the temporal evolution of the mean kinetic energy and enstrophy is presented in the Fig. 5. In order to provide a quantitative comparison between different schemes, we calculate accuracy metrics for the Taylor-Green vortex following [61]. The mean kinetic energy normalized by its initial value at $t=5$ and the mean enstrophy normalized by its initial value at $t=3.5$ are calculated respectively. The results from different scheme are presented in Table 1. These results of inviscid Taylor-Green vortex show that $\mathrm{P}_{n} \mathrm{~T}_{m}-\mathrm{BVD}$ schemes have overall better accuracy than WENOM schemes. Although the numerical dissipation is limited by upwind-biased interpolations for all schemes, the $\mathrm{P}_{n} \mathrm{~T}_{m}-\mathrm{BVD}$ are still much less dissipative than WENOM. It should be noted that schemes which introduce non-dissipative central flux can exactly preserve the discrete total kinetic energy $[8,60,62]$.

\subsection{Taylor-Green vortex problem with $R e=1600$}

The three-dimensional periodic Taylor-Green vortex is simulated in this subsection. Taylor-Green vortex serves as a canonical problem which contains key physical processes in turbulence such as turbulent transition, turbulent decay and the energy dissipation process. Thus it is usually employed to evaluate the method for turbulent flow simulation. The problem consists of a cubic volume of side length $2 \pi L$ with an analytic initial condition defined by

$$
\begin{aligned}
u & =U_{0} \sin (x / L) \cos (y / L) \cos (z / L), \\
v & =-U_{0} \cos (x / L) \sin (y / L) \cos (z / L), \\
w & =0, \\
p & =p_{0}+\frac{\rho_{0} U_{0}^{2}[\cos (2 x / L)+\cos (2 y / L)][\cos (2 z / L)+2]}{16}
\end{aligned}
$$

where $L=1.0, U_{0}=1.0, \rho_{0}=1.0$ and $p_{0}=100.0$. The initial temperature is $300 \mathrm{k}$. The Mach number is set to $M_{0}=\frac{U_{0}}{c_{0}}=$ 0.08 and the Reynolds number is set to $R e_{0}=\frac{\rho_{0} U_{0} L}{\mu}=1600$ by adjusting the viscosity. We use the characteristic convective time defined as $t=\frac{L}{U_{0}}$. A coarse mesh consisting of $64^{3}$ elements is employed in our calculation. In order to evaluate the 
a

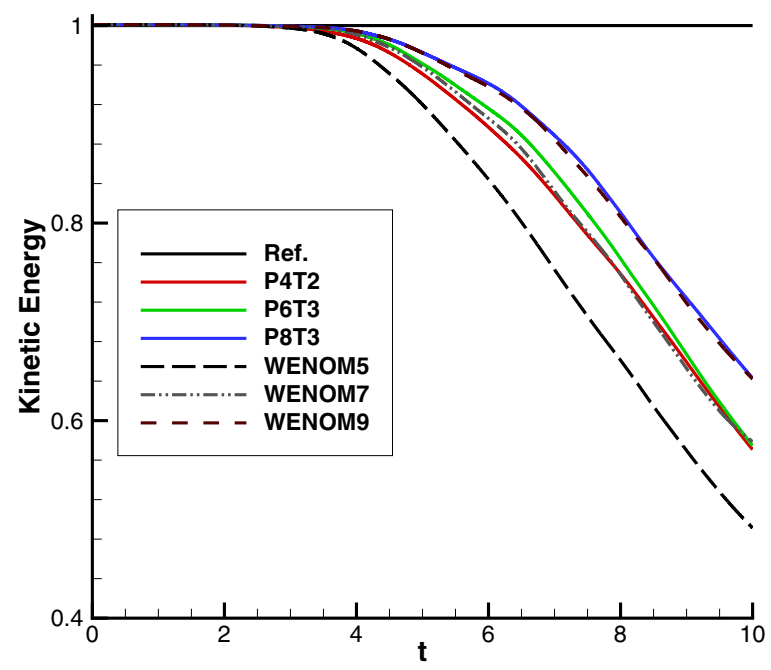

b

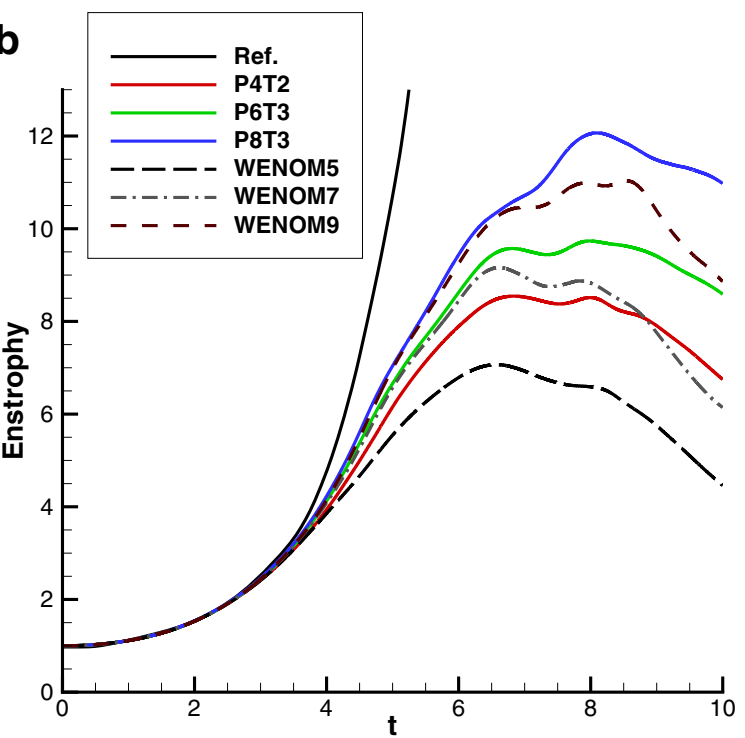

Fig. 5. Evolution of mean kinetic energy and enstrophy for the inviscid Taylor-Green vortex problem. Comparisons between $P_{n} T_{m}-B V D$ and WENOM schemes are presented. The evolution for the mean kinetic energy is shown in (a) and for the enstrophy is shown in (b).

Table 1

Accuracy metrics for the Taylor-Green vortex. Comparisons between different schemes and the semi-analytical result of [61].

\begin{tabular}{llllllll}
\hline & WENOM5 & WENOM7 & WENOM9 & $\mathrm{P}_{4} \mathrm{~T}_{2}-$ BVD & $\mathrm{P}_{6} \mathrm{~T}_{3}-$ BVD & $\mathrm{P}_{8} \mathrm{~T}_{3}-$ BVD & Ref. [61] \\
\hline T-G energy $t=5$ & 0.922 & 0.957 & 0.971 & 0.950 & 0.960 & 0.972 & 1 \\
T-G enstrophy $t=3.5$ & 3.082 & 3.153 & 3.170 & 3.083 & 3.160 & 3.207 & 3.46 \\
\hline
\end{tabular}

performance of schemes, we introduce the diagnostic quantities include the integrated kinetic energy $E_{k}$ and the kinetic energy dissipation rate $\epsilon_{k}$, which are defined as

$$
E_{k}=\frac{1}{\Delta V} \int \frac{1}{2} \rho\left(u^{2}+v^{2}+w^{2}\right) d V, \quad \epsilon\left(E_{k}\right)=-\frac{d E_{k}}{d t}
$$

We conduct the simulation with the newly proposed $\mathrm{P}_{n} \mathrm{~T}_{m}-$ BVD schemes and the WENOM schemes of 5th, 7th and 9th order.

Firstly, we present the temporal evolution of integrated kinetic energy simulated with different schemes in Fig. 6. The reference solution is obtained by high-resolution DNS simulation on a mesh of $512^{3}$ [45]. As a whole, the solution of 5th order WENOM scheme (black dashed line) largely deviates with the reference solution. The inherent numerical dissipation of 5th order WENOM leads to rapid decay of kinetic energy. By increasing the order, 7th and 9th order WENOM (grey and purple dashed line) schemes produce solution closer to the reference one. Compared with WENOM, $\mathrm{P}_{n} \mathrm{~T}_{m}-\mathrm{BVD}$ schemes show better performance. For example, 5th order $\mathrm{P}_{4} \mathrm{~T}_{2}-\mathrm{BVD}$ (red line) scheme producing competitive solution with 7 th order WENOM. 7th order $\mathrm{P}_{6} \mathrm{~T}_{3}-\mathrm{BVD}$ and 9th order $\mathrm{P}_{8} \mathrm{~T}_{3}-\mathrm{BVD}$ (green and blue line) obtain the most accurate results compared to the reference solution with $\mathrm{P}_{8} \mathrm{~T}_{3}-\mathrm{BVD}$ produces a little better one. It should be noted that during $10<t<20$, the kinetic energy decay speeds of WENOM7 and WENOM9 become slower than the reference one and result in overestimated kinetic energy at final time. However, 7th order and 9th order BVD schemes accurately predict the kinetic energy even after long temporal evolution.

To further evaluate the performance of schemes, the evolution of kinetic energy dissipation rate with $\mathrm{P}_{n} \mathrm{~T}_{m}-\mathrm{BVD}$ is plotted in Fig. 7. With order increased, the prediction of the evolution becomes more accurate in terms of the evolution trend and the peak of the dissipation rate. The comparisons between WENOM schemes of different orders are presented in Fig. 8. It is obvious that BVD schemes produce overall better results. For example, in Fig. 8 (b) the 5th order $\mathrm{P}_{4} \mathrm{~T}_{2}-\mathrm{BVD}$ produces competitive solution compared with 7th order WENOM, and in Fig. 8 (c) the 7th order $\mathrm{P}_{6} \mathrm{~T}_{3}-$ BVD even obtains a little better solution compared with 9th order WENOM regarding to prediction of the peak of the dissipation rate.

To show the influence of different wavenumber on the decay of the turbulent structures, the spectra of turbulent kinetic energy at $t=9$ is shown in Fig. 9 with the reference spectral solution [63]. Again, $\mathrm{P}_{n} \mathrm{~T}_{m}-\mathrm{BVD}$ schemes show better results compared with WENOM. The advantages of $\mathrm{P}_{n} \mathrm{~T}_{m}-\mathrm{BVD}$ schemes become more obvious as the wave number increases. As shown in Fig. 9(b), all of WENOM schemes are inferior to the $\mathrm{P}_{n} \mathrm{~T}_{m}-$ BVD schemes in high wavenumber region. In a 
a

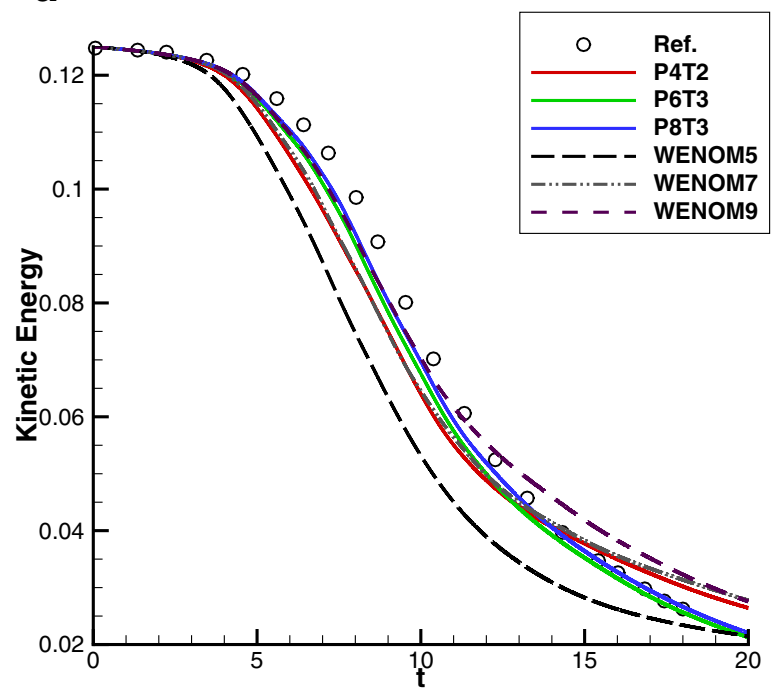

b

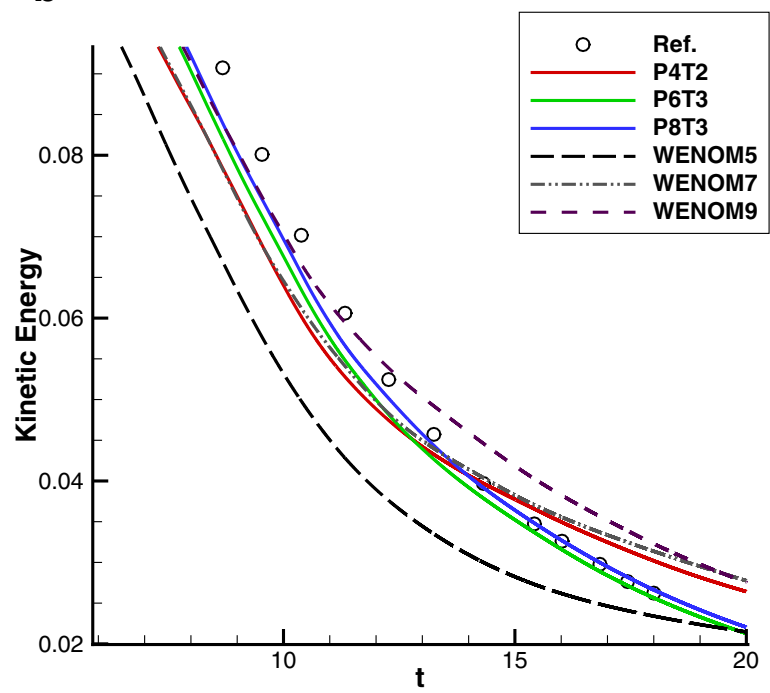

Fig. 6. Evolution of mean kinetic energy for the Taylor-Green vortex problem. Comparisons between $\mathrm{P}_{n} \mathrm{~T}_{m}-\mathrm{BVD}$ and WENOM schemes are presented. The evolution for the whole time band is shown in (a) and the zoomed region for the later time is shown in (b).

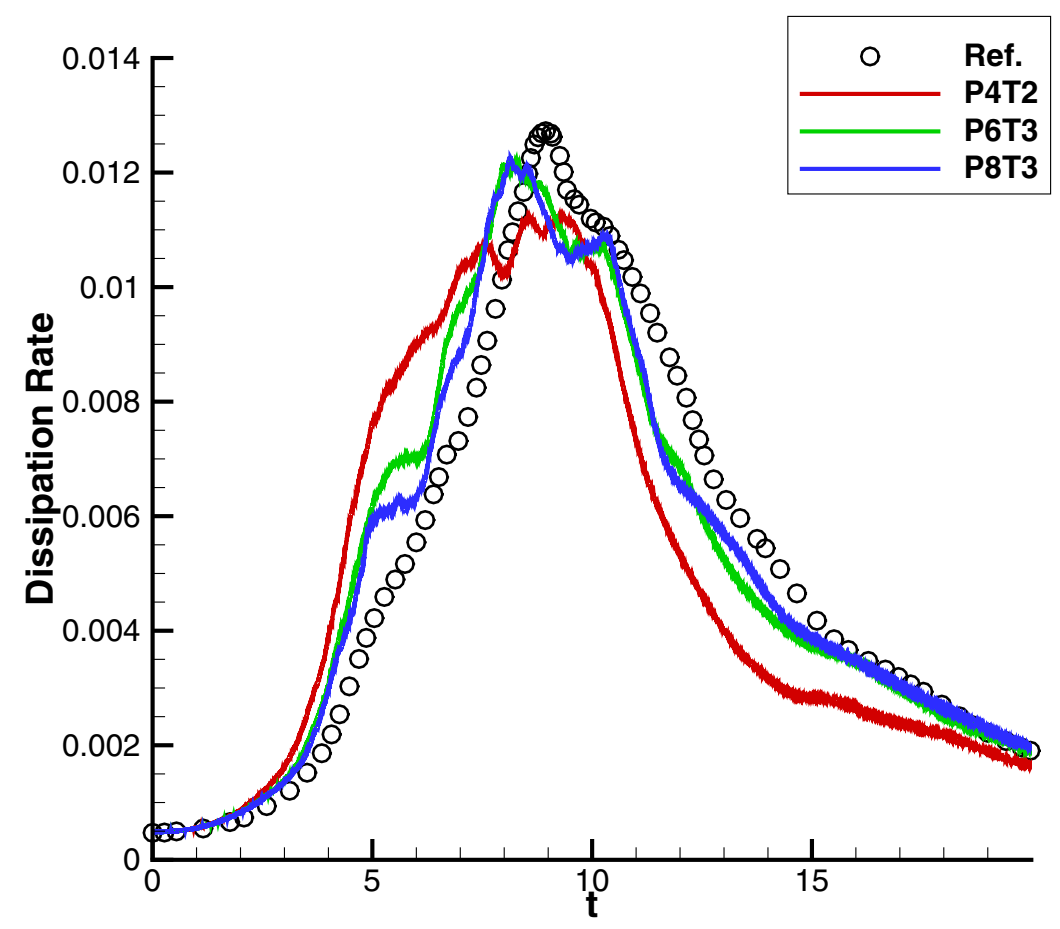

Fig. 7. Temporal evolution of kinetic energy dissipation rate for the Taylor-Green vortex problem. Results are calculated by $\mathrm{P}_{n} \mathrm{~T}_{m}-\mathrm{BVD}$ of different orders.

conclusion of above analysis, $\mathrm{P}_{n} \mathrm{~T}_{m}-\mathrm{BVD}$ schemes produce high resolution and high fidelity solution for the Taylor-Green vortex simulation.

\subsection{Compressible isotropic turbulence}

The decaying compressible isotropic turbulence with eddy shocklets [8] is simulated in this subsection. In this problem, weak shock waves will develop from the turbulent motions if a sufficiently high turbulent Mach number is provided. Thus the coexist of shock waves as well as turbulence requires the shock-capturing scheme should be able to handle shocklets and 
a

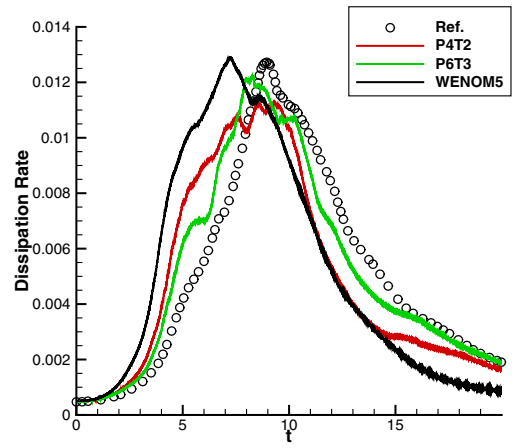

b

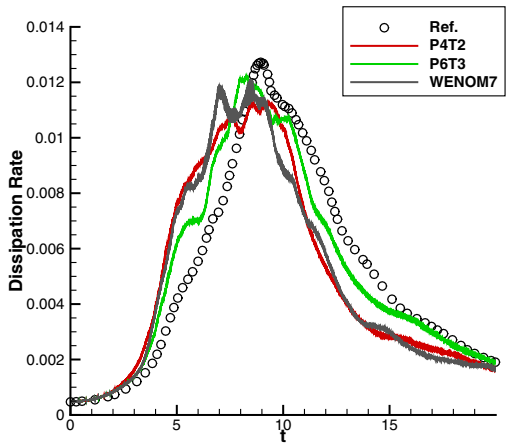

C

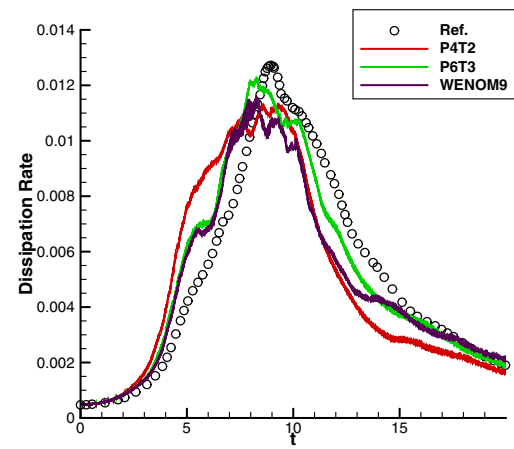

Fig. 8. Temporal evolution of kinetic energy dissipation rate for the Taylor-Green vortex problem. Comparison between $P_{n} T_{m}-B V D$ with 5 -th order WENOM in (a), with 7-th order WENOM in (b) and with 9-th order WENOM in (c).

a

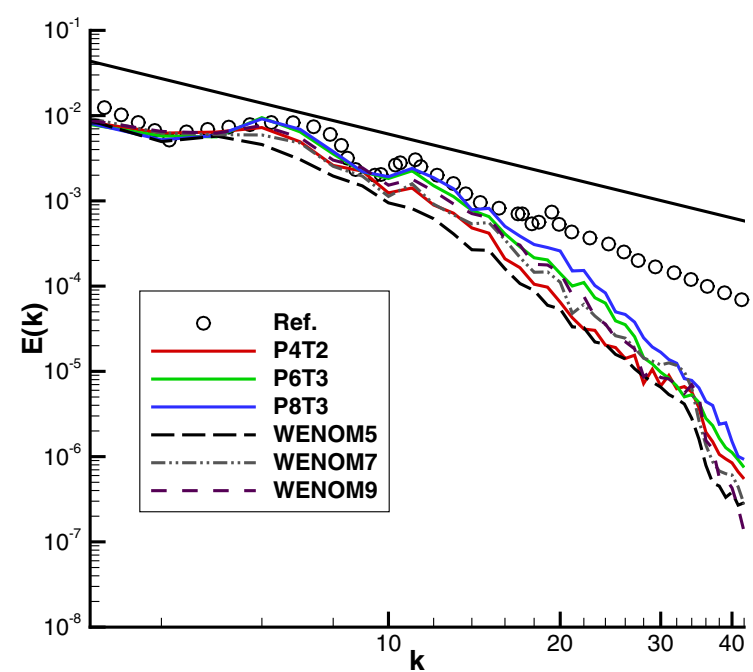

b

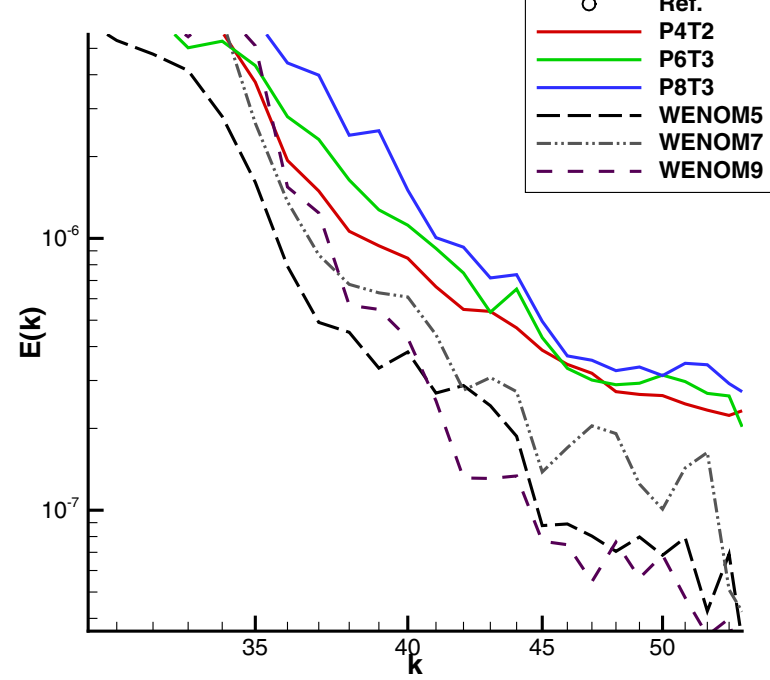

Fig. 9. Spectra of turbulent kinetic energy at $t=9$. Comparisons between $\mathrm{P}_{n} \mathrm{~T}_{m}-\mathrm{BVD}$ and WENOM schemes. The evolution for the whole wavenumber is shown in (a) and the zoomed region for the high wavenumber is shown in (b).

simultaneously resolve broadband turbulence motion. The computational domain and the boundary conditions are the same as those with the Taylor-Green vortex case. A coarse mesh consisting of $64^{3}$ elements is also employed in our calculation to conduct ILES. The initial condition consists of a random isotropic velocity field velocity fluctuations satisfying a prescribed energy spectrum as

$$
E_{k}=u_{r m s, 0}^{2} 16 \sqrt{\frac{2}{\pi}} \frac{k^{4}}{k_{0}^{5}} \exp \left(-\frac{2 k^{2}}{k_{0}^{2}}\right)
$$

where $u_{r m s, 0}$ is the root mean square turbulence intensity and the wavenumber $k_{0}=4$. The initial turbulent Mach number and Taylor-scale Reynolds number are defined as

$$
M_{t, 0}=\sqrt{3} u_{r m s, 0} / c_{0}, \quad R e_{\lambda, 0}=\rho_{0} u_{r m s, 0} \lambda_{0} / \mu_{0}
$$

where $\lambda_{0}=\frac{2}{k_{0}}$ and $\mu_{0}$ is initial dynamic viscosity based on the initial temperature $T_{0}$. The viscosity is assumed to follow a power law. Initially, the density and pressure are constant over the domain, which are prescribed with $M_{t, 0}=0.6$ and $R e_{\lambda, 0}=100$. The problem is solved till a final time of $t / \tau=4$ where $\tau=\lambda / u_{r m s, 0}$ is the turbulent time scale. According to [], this initial condition will produce weak shock waves and broadband turbulence flows.

In order to evaluate the performance of the numerical schemes, the temporal evolution of the mean-square velocity, and the variance of the temperature and dilatation are presented in Figs. 10, 11 and 12, respectively. The solutions calculated by $\mathrm{P}_{4} \mathrm{~T}_{2}-\mathrm{BVD}, \mathrm{P}_{6} \mathrm{~T}_{3}-\mathrm{BVD}$ and $\mathrm{P}_{8} \mathrm{~T}_{3}-\mathrm{BVD}$ are plotted with red, green and blue solid lines respectively. To make a comparison, we also include the results calculated with 5th-, 7th- and 9th- order WENOM schemes which are marked by red, 
a

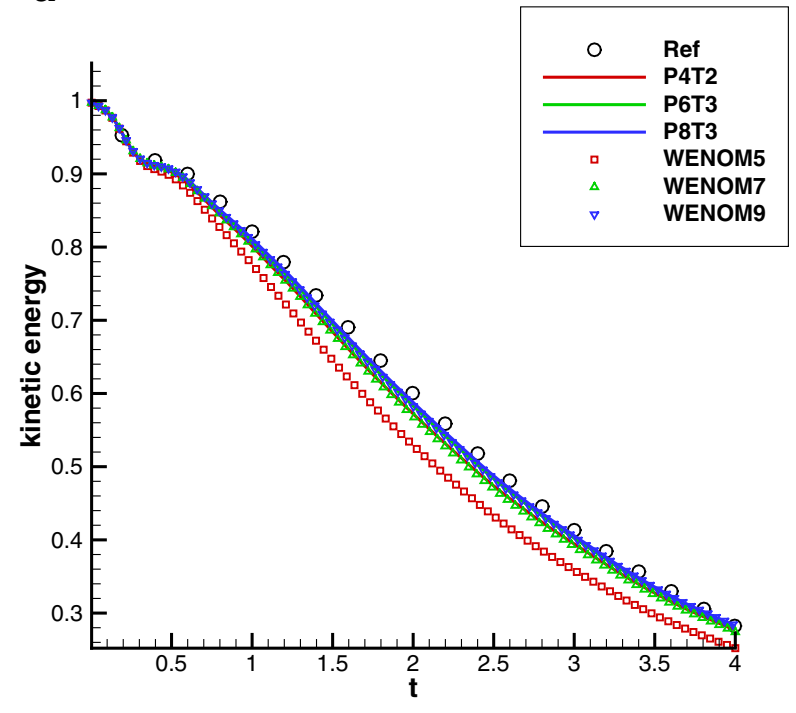

b

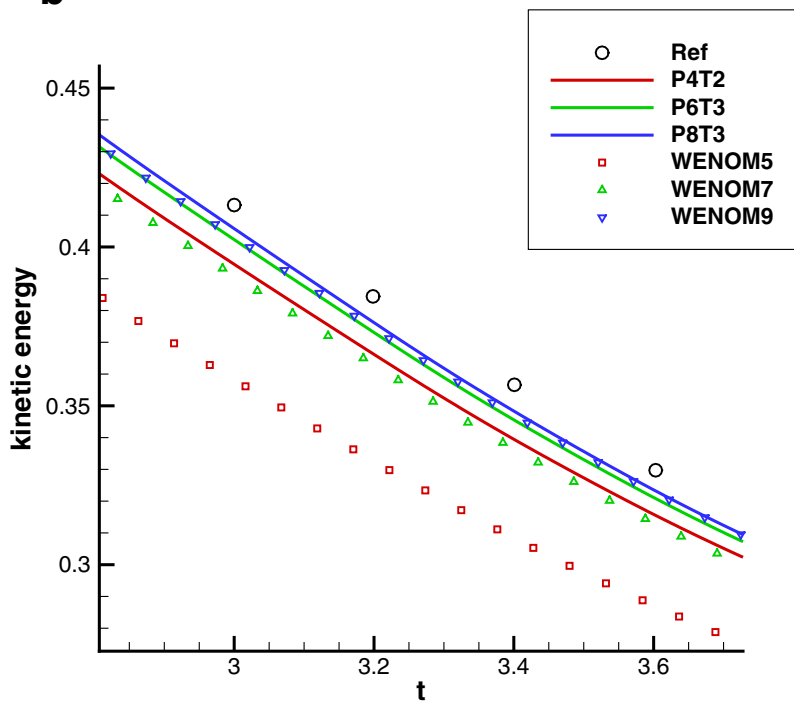

Fig. 10. Evolution of mean-square velocity for the compressible isotropic turbulence. Comparisons between $\mathrm{P}_{n} \mathrm{~T}_{m}-\mathrm{BVD}_{\mathrm{B}}$ and WENOM schemes are presented. The evolution for the whole time band is shown in (a) and the zoomed region for long time evolution is shown in (b).

a

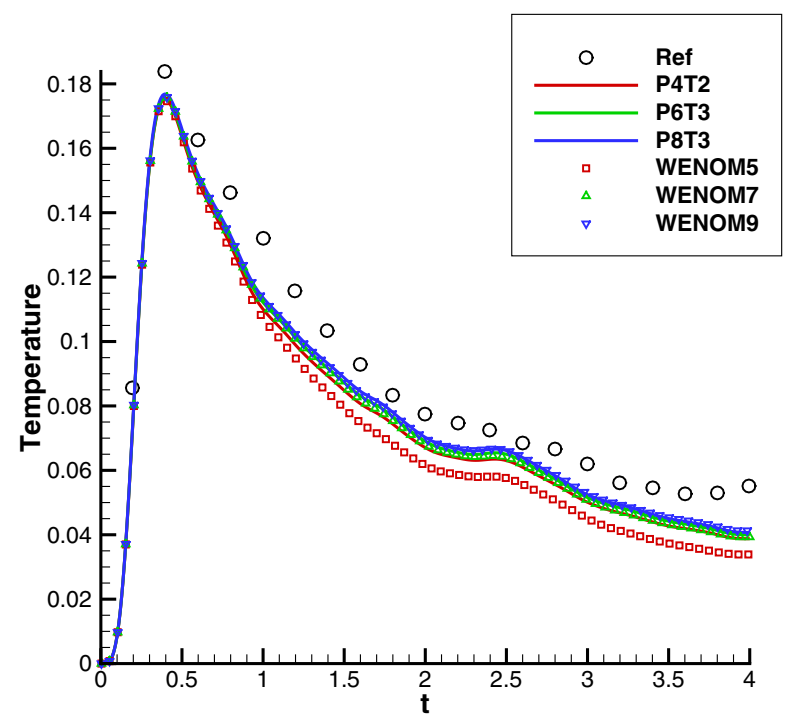

b

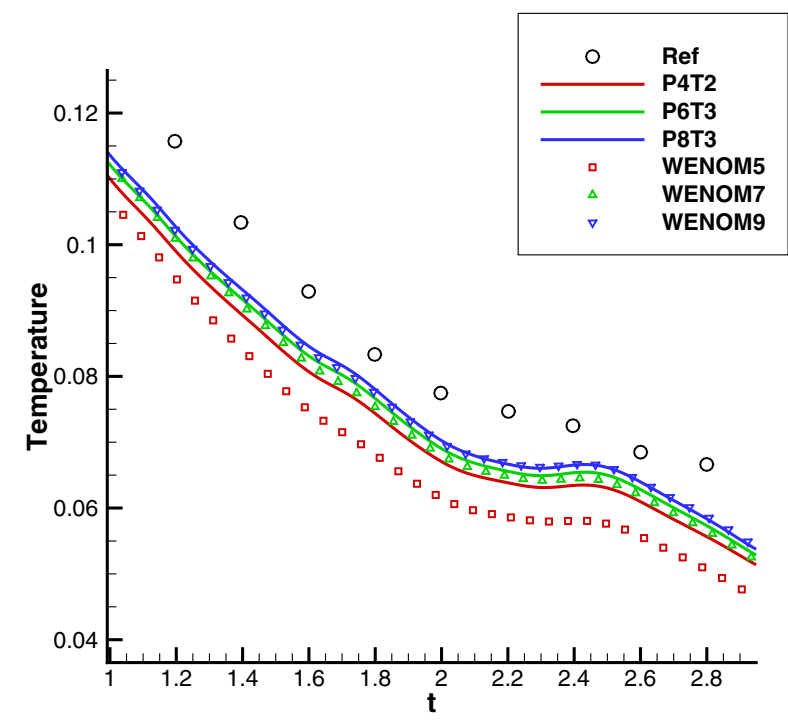

Fig. 11. Evolution of the temperature for the compressible isotropic turbulence. Comparisons between $\mathrm{P}_{n} \mathrm{~T}_{m}-\mathrm{BVD}$ and WENOM schemes are presented. The evolution for the whole time band is shown in (a) and the zoomed region is shown in (b).

green and blue symbols respectively. Again, the $\mathrm{P}_{n} \mathrm{~T}_{m}-\mathrm{BVD}$ schemes produce superior results compared with WENOM. For example, the 5th order $\mathrm{P}_{4} \mathrm{~T}_{2}-$ BVD scheme obtain solutions which are comparable or even better than 7th order WENOM. Similarly, the 7th order $\mathrm{P}_{4} \mathrm{~T}_{2}-$ BVD scheme is competitive with 9 th order WENOM. Thus, $\mathrm{P}_{n} \mathrm{~T}_{m}-\mathrm{BVD}$ is more accurate and less diffusive.

We also plot the velocity spectrum at $t / \tau=4$ along with the reference solution [8] in Fig. 13. Over lower wavenumbers, all schemes seem to produce similar results. However, advantages of $\mathrm{P}_{n} \mathrm{~T}_{m}-\mathrm{BVD}$ become notable over the higher wavenumber band. For example, the 7 th order $\mathrm{P}_{n} \mathrm{~T}_{m}-$ BVD get closer solution to the reference than 9th order WENOM in high wavenumber region.

Finally, the convergence test for the compressible isotropic turbulence problem has been performed on different grid resolutions to see if fully converged DNS results can be achieved with our ILES results. The comparison is made similar 
a

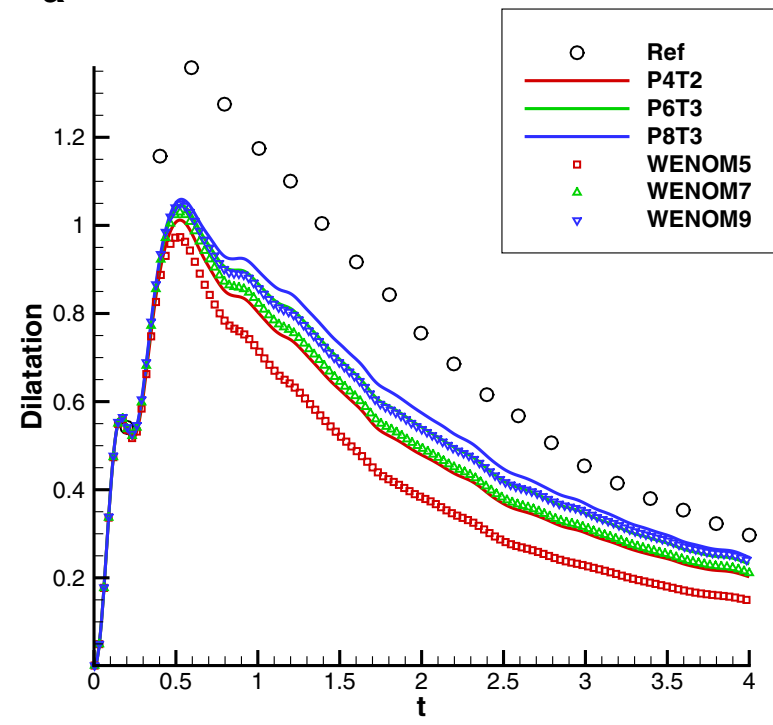

b

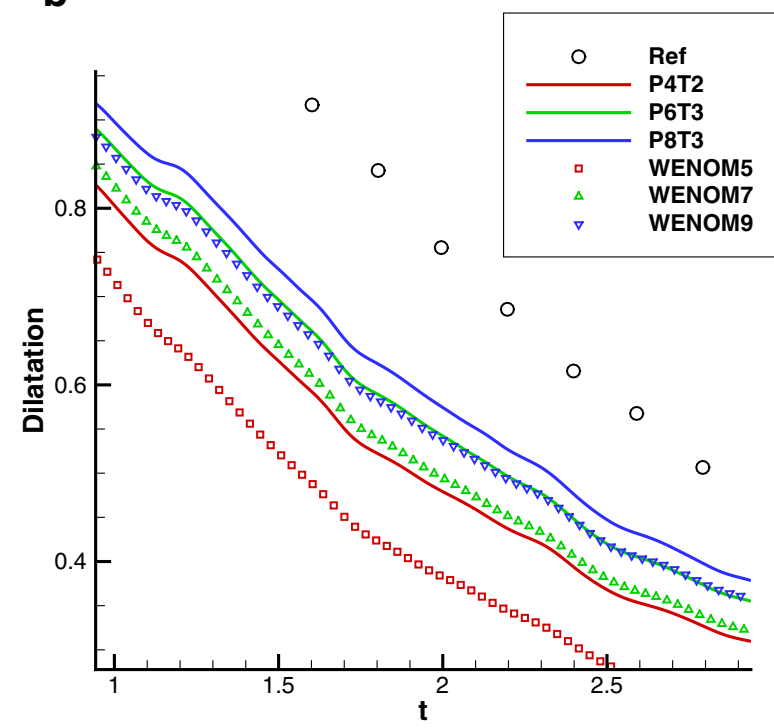

Fig. 12. Evolution of the dilatation for the compressible isotropic turbulence. Comparisons between $P_{n} T_{m}-B V D$ and WENOM schemes are presented. The evolution for the whole time band is shown in (a) and the zoomed region is shown in (b).

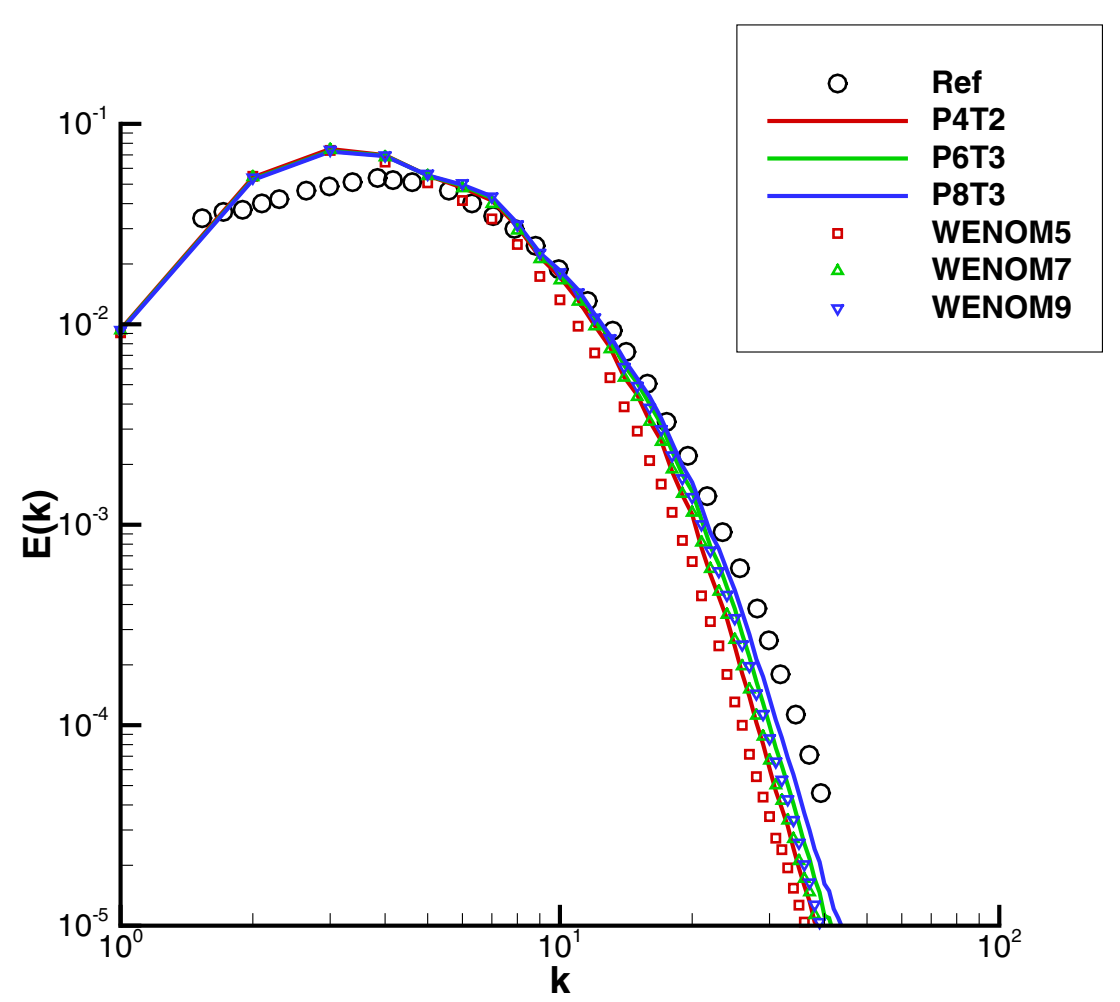

Fig. 13. Velocity spectra for the isotropic turbulence problem at $t / \pi=4$. Comparisons between $\mathrm{P}_{n} \mathrm{~T}_{m}-\mathrm{BVD}_{\mathrm{and}} \mathrm{WENOM}$ schemes on $64^{3}$ grids.

to the one in [23]. Here the turbulence kinetic energy spectra is employed to validate the convergence of the $\mathrm{P}_{n} \mathrm{~T}_{m}-\mathrm{BVD}$ schemes. The numerical results of different order schemes are presented in the Fig. 14. It can be seen obviously that the grid-converged spectrum can be reached when the number of the grid elements is over $128^{3}$. And the higher order schemes show faster convergence in comparison with the DNS solution. 


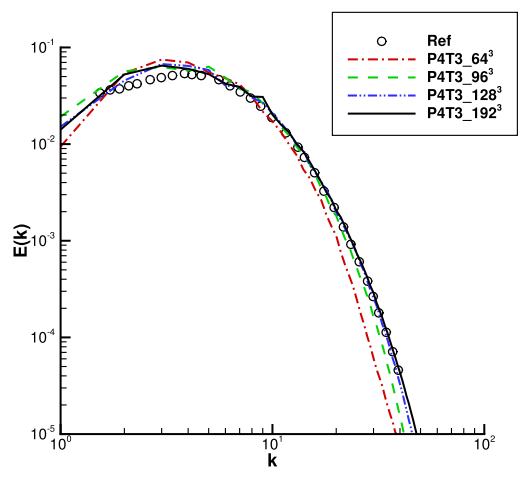

(a) $\mathrm{P}_{4} \mathrm{~T}_{2}-\mathrm{BVD}$

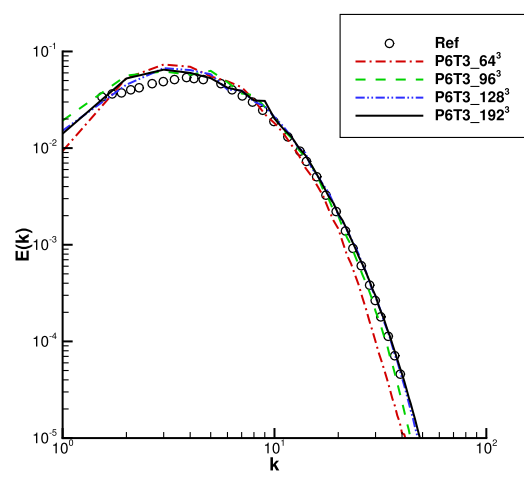

(b) $\mathrm{P}_{6} \mathrm{~T}_{3}-\mathrm{BVD}$

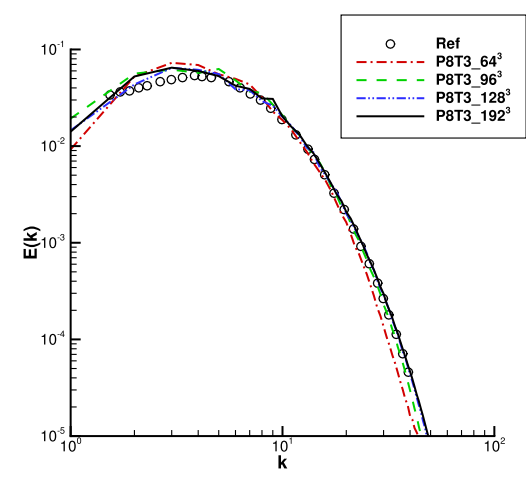

(c) $\mathrm{P}_{8} \mathrm{~T}_{3}-\mathrm{BVD}$

Fig. 14. Convergence test for compressible isotropic turbulence problem on different grid resolutions. Comparison between DNS results with 5-th order $\mathrm{P}_{4} \mathrm{~T}_{2}-\mathrm{BVD}$ in (a), with 7-th order $\mathrm{P}_{6} \mathrm{~T}_{3}-\mathrm{BVD}$ in (b) and with 9-th order $\mathrm{P}_{8} \mathrm{~T}_{3}-\mathrm{BVD}$ in (c).

\section{Concluding remarks}

A newly proposed $\mathrm{P}_{n} \mathrm{~T}_{m}$ - BVD schemes are implemented to compressible Navier-Stokes equations to evaluate their performance in ILES of turbulent compressible flows. Compared with high order WENOM, $\mathrm{P}_{n} \mathrm{~T}_{m}-\mathrm{BVD}$ schemes have following two obvious advantages, 1) they can retrieve the unlimited high order polynomial reconstructions in all wave-number region, and 2) they can obtain adequately robust results and effectively suppress numerical oscillations in the presence of strong discontinuities. Moreover, simulations of Taylor-Green vortex and compressible isotropic turbulence show that $\mathrm{P}_{n} \mathrm{~T}_{m}$ - BVD schemes are able to give more accurate prediction of the turbulence evolution in terms of the evolution trend and the peak of the dissipation rate. Importantly, the superiority of $\mathrm{P}_{n} \mathrm{~T}_{m}$ - BVD becomes more notable in high wave-number region. Thus, the newly proposed $\mathrm{P}_{n} \mathrm{~T}_{m}$ - BVD scheme can serve as an alternative method which provides high resolution solution for ILES of compressible turbulence.

It's noteworthy that the $\mathrm{P}_{n} \mathrm{~T}_{m}$ - BVD framework provides a limiting process which preserves the spectral property of the underlying high order interpolation for all wavenumber, which indicates the numerical dissipation introduced from the limiting process has been effectively controlled. However, the present scheme still employs simple upwind-biased interpolation functions as the underlying high order schemes, which is now the main source of numerical errors. In our future work, we will optimize and control the numerical dissipation by improving the underlying interpolation function with the methods employed in $[22,23,49]$. With optimized underlying high order interpolation, it has great potential to achieve better results for ILES of compressible turbulence flow.

\section{Acknowledgment}

This work was supported in part by the fund from JSPS (Japan Society for the Promotion of Science) under Grant Nos. $17 \mathrm{~K} 18838$ and 18H01366, and also in part by the National Natural Science Foundation of China (grants 11702015).

\section{References}

[1] A. Harten, High resolution schemes for hyperbolic conservation laws, J. Comput. Phys. 49 (1983) 357-393.

[2] P.K. Sweby, High resolution schemes using flux-limiters for hyperbolic conservation laws, SIAM J. Num. Anal. 21 (1984) $995-1011$.

[3] A. Harten, B. Engquist, S. Osher, S. Chakravarthy, Uniformly high order accurate essentially non-oscillatory schemes III, J. Comput. Phys. 71 (1987) $231-323$.

[4] C.W. Shu, S. Osher, Efficient implementation of essentially non-oscillatory shock capturing schemes, J. Comput. Phys. 77 (1988) $439-471$.

[5] C.W. Shu, S. Osher, Efficient implementation of essentially non-oscillatory shock capturing schemes, II, J. Comput. Phys. 83 (1989) $32-78$.

[6] X.D. Liu, S. Osher, T. Chan, Weighted essentially non-oscillatory schemes, J. Comput. Phys. 115 (1994) 200-212.

[7] G.S. Jiang, C.W. Shu, Efficient implementation of weighted ENO schemes, J. Comput. Phys. 126 (1996) 202-228.

[8] E. Johnsen, J. Larsson, A.V. Bhagatwala, et al., Assessment of high-resolution methods for numerical simulations of compressible turbulence with shock waves, J. Comput. Phys. 229 (4) (2010) 1213-1237.

[9] F. Ducros, F. Laporte, T. Souleres, V. Guinot, P. Moinat, B. Caruelle, High-order fluxes for conservative skew-symmetric-like schemes in structured meshes: application to compressible flows, J. Comput. Phys. 161 (2000) 114-139.

[10] D.J. Hill, D.I. Pullin, Hybrid tuned center-difference-WENO method for large eddy simulations in the presence of strong shocks, J. Comput. Phys. 194 (2004) 435-450.

[11] J. Larsson, B. Gustafsson, Stability criteria for hybrid difference methods, J. Comput. Phys. 227 (2008) 2886-2898.

[12] D. Kim, H.K. Kwon, A high-order accurate hybrid scheme using a central flux scheme and a WENO scheme for compressible flowfield analysis, J. Comput. Phys. 210 (2005) 554-583.

[13] S. Pirozzoli, Conservative hybrid compact-WENO schemes for shock-turbulence interaction, J. Comput. Phys. 178 (2002) 81-117.

[14] A.W. Cook, Artificial properties for large-eddy simulation of compressible turbulent mixing, Phys. Fluids 19 (2007) 055103.

[15] A.W. Cook, W.H. Cabot, A high-wavenumber viscosity for high-resolution numerical methods, J. Comput. Phys. 195 (2004) 594-601.

[16] A.W. Cook, W.H. Cabot, Hyperviscosity for shock-turbulence interactions, J. Comput. Phys. 203 (2005) 379-385.

[17] B. Fiorina, S.K. Lele, An artificial nonlinear diffusivity method for supersonic reacting flows with shocks, J. Comput. Phys. 222 (2007) $246-264$. 
[18] V. Daru, C. Tenaud, High order one-step monotonicity-preserving schemes for unsteady compressible flow calculations, J. Comput. Phys. 193 (2004) $563-594$.

[19] W.J. Rider, L.G. Margolin, Simple modifications of monotonicity-preserving limiter, J. Comput. Phys. 174 (2001) 473-488.

[20] A. Suresh, H.T. Huynh, Accurate monotonicity-preserving schemes with Runge-Kutta time stepping, J. Comput. Phys. 136 (1997) 83-99.

[21] G.H. Tu, X.J. Yuan, A characteristic-based shock-capturing scheme for hyperbolic problems, J. Com- put. Phys. 225 (2007) $2083-2097$.

[22] X. Li, Y. Leng, Z. He, Optimized sixth-order monotonicity-preserving scheme by nonlinear spectral analysis, Int. J. Numer. Meth. Fluids. 73 (2013) $560-577$.

[23] J. Fang, Z. Li, L. Lu, An optimized low-dissipation monotonicity-preserving scheme for numerical simulations of high-speed turbulent flows, J. Sci. Comput. 56 (2013) 67-95.

[24] B. Cockburn, S. Hou, C.W. Shu, The runge-kutta local projection discontinuous galerkin finite element method for conservation laws. IV, The multidimensional case, Math. Comput. 54 (1990) 545-581.

[25] F. Bassi, S. Rebay, High-order accurate discontinuous finite element solution of the 2d euler equations, J. Comput. Phys. 138 (1997) 251-285.

[26] P.E. Vincent, P. Castonguay, A. Jameson, A new class of high-order energy stable flux reconstruction schemes, J. Scient. Comput. 47 (2011) 50-72.

[27] M. Dumbser, O. Zanotti, R. Loubere, S. Diot, A posteriori subcell limiting of the discontinuous Galerkin finite element method for hyperbolic conservation laws, J. Comput. Phys. 278 (2014) 47-75.

[28] J. Qiu, C.W. Shu, A comparison of troubled-cell indicators for Runge-Kutta discontinuous Galerkin methods using weighted essentially nonoscillatory limiters, SIAM J. Sci. Comput. 27 (2005) 995-1013.

[29] L. Krivodonova, Limiters for high-order discontinuous Galerkin methods, J. Comput. Phys. 226 (2007) $879-896$.

[30] X. Deng, B. Xie, F. Xiao, A finite volume multi-moment method with boundary variation diminishing principle for euler equation on three-dimensional hybrid unstructured grids, Comput. Fluids 153 (2017) 85-101.

[31] F.F. Grinstein, L.G. Margolin, W.J. Rider, Implicit Large Eddy Simulation: computing Turbulent Fluid Dynamics, Cambridge university press, 2007.

[32] J. Boris, F.F. Grinstein, E. Oran, R. Kolbe, New insights into large eddy simulation, Fluid Dyn. Res. 10 (1992) 199-228.

[33] Y. Cao, T. Tamura, Large-eddy simulations of flow past a square cylinder using structured and unstructured grids, Comput. Fluids 137 (2016) 36-54.

[34] K. Ritos, I.W. Kokkinakis, D. Drikakis, S.M. Spottswood, Implicit large eddy simulation of acoustic loading in supersonic turbulent boundary layers, Phys. Fluids 29 (2017) 1-11.

[35] J.A. Domaradzki, S. Radhakrishnan, Effective eddy viscosities in implicit modeling of decaying high reynolds number turbulence with and without rotation, Fluid Dyn. Res. 36 (2005) 385-406.

[36] K. Ritos, I.W. Kokkinakis, D. Drikakis, Performance of high-order implicit large eddy simulations, Comput. Fluids 173 (2018) $307-312$.

[37] E. Garnier, M. Mossi, P. Sagaut, P. Comte, M. Deville, On the use of shock-capturing schemes for large-eddy simulation, J. Comput. Phys. 153 (1999) $273-311$.

[38] S. Zhao, N. Lardjane, I. Fedioun, Comparison of improved finite-difference WENO schemes for the implicit large eddy simulation of turbulent non-reacting and reacting high-speed shear flows, Comput. Fluids 95 (2014) 74-87.

[39] Z. Sun, S. Inaba, F. Xiao, Boundary variation diminishing (BVD) reconstruction: a new approach to improve godunov schemes, J. Comput. Phys. 322 (2016) 309-325.

[40] X. Deng, S. Inaba, B. Xie, K.M. Shyue, F. Xiao, High fidelity discontinuity-resolving reconstruction for compressible multiphase flows with moving interfaces, J. Comput. Phys. 371 (2018) 945-966.

[41] X. Deng, B. Xie, F. Xiao, H. Teng, New accurate and efficient method for stiff detonation capturing, AIAA J. (2018) 1-15.

[42] X. Deng, B. Xie, R. Loubère, Y. Shimizu, F. Xiao, Limiter-free discontinuity-capturing scheme for compressible gas dynamics with reactive fronts, Comput. Fluids 171 (2018) 1-14.

[43] X. Deng, Y. Shimizu, F. Xiao, A fifth-order shock capturing scheme with two-stage boundary variation diminishing algorithm, J. Comput. Phys. 386 (2019) 323-349.

[44] X. Deng, Y. Shimizu, F. Xiao, Constructing high-order discontinuity-capturing schemes with linear-weight polynomials and boundary variation diminishing algorithm. 2018, arXiv preprint arXiv:1811.08316.

[45] J.R. DeBonis, Solutions of the Taylor-Green Vortex Problem Using High Resolution Explicit Finite Difference Methods, AIAA Paper 2013-0382, 2013.

[46] D.S. Balsara, C.W. Shu, Monotonicity preserving WENO schemes with increasingly high-order of accuracy, J. Comput. Phys. 160 (2000) 405-452.

[47] F. Xiao, S. Ii Ii, C. Chen, Revisit to the THINC scheme: a simple algebraic VOF algorithm, J. Comput. Phys. 230 (2011) $7086-7092$.

[48] F. Xiao, Y. Honma, T. Kono, A simple algebraic interface capturing scheme using hyperbolic tangent function, Int. J. Numer. Methods Fluids 48 (2005) $1023-1040$.

[49] L. Fu, X.Y. Hu, N.A. Adams, A family of high-order targeted ENO schemes for compressible-fluid simulations, J. Comput. Phys. 305 (2016) 333-359,

[50] A.K. Henrick, T.D. Aslam, J.M. Powers, Mapped weighted essentially non-oscillatory schemes: achieving optimal order near critical points, J. Comput. Phys. 207 (2005) 542-567.

[51] R. Borges, M. Carmona, B. Costa, W.S. Don, An improved weighted essentially non-oscillatory scheme for hyperbolic conservation laws, J. Comput. Phys. 227 (2008) 3191-3211.

[52] S. Pirozzoli, On the spectral properties of shock-capturing schemes, J. Comput. Phys. 219 (2006) $489-497$.

[53] H.H. Teng, Z.L. Jiang, On the transition pattern of the oblique detonation structure, J. Fluid Mech. 713 (2012) 659-669.

[54] H.H. Teng, Z.L. Jiang, H.D. Ng, Numerical study on unstable surfaces of oblique detonations, J. Fluid Mech. 744 (2014) $111-128$.

[55] H.H. Teng, H.D. Ng, K. Li, C.T. Luo, Z.L. Jiang, Evolution of cellular structures on oblique detonation surfaces, Combust. Flame 162 (2015) 470-477.

[56] H.H. Teng, H.D. Ng, Z.L. Jiang, Initiation characteristics of wedge-induced oblique detonation waves in a stoichiometric hydrogen-air mixture, Proc. Combust. Inst. 36 (2017) 2735-2742.

[57] P. Yang, H.H. Teng, Z.L. Jiang, H.D. Ng, Effects of inflow mach number on oblique detonation initiation with a two-step induction-reaction kinetic model, Combust. Flame 193 (2018) 246-256.

[58] Y. Zhang, Y. Fang, H.D. Ng, H.H. Teng, Numerical investigation on the initiation of oblique detonation waves in stoichiometric acetylene-oxygen mixtures with high argon dilution, Combust. Flame 204 (2019) 391-396.

[59] V.A. Titarev, E.F. Toro, Finite-volume WENO schemes for three-dimensional conservation laws, J. Comput. Phys. 201 (2004) $238-260$.

[60] D. Modesti, S. Pirozzoli, A low-dissipative solver for turbulent compressible flows on unstructured meshes with openFOAM implementation, Comput. Fluids 152 (2017) 14-23.

[61] M.E. Brachet, D.I. Meiron, S.A. Orszag, B.G. Nickel, R.H. Morf, U. Frisch, Small-scale structure of the Taylor-Green vortex, J. Fluid Mech. 130 (1983) $411-452$.

[62] S. Pirozzoli, Generalized conservative approximations of split convective derivative operators, J. Comput. Phys. 229 (2010) $7180-7190$.

[63] C.C. de Wiart, K. Hillewaert, M. Duponcheel, G. Winckelmans, Assessment of a discontinuous Galerkin method for the simulation of vortical flows at high Reynolds number, Int. J. Numer. Methods Fluids 74 (2014) 469-493. 